\title{
Dinámica del fitoplancton en tres lagos urbanos con diferentes estrategias de manejo: Análisis de floraciones estivales
}

\author{
Clara N. Rodríguez-FlóreZ; Alicia Vinocur ${ }^{2,3} \&$ Irina Izaguirre ${ }^{2,}$, \\ ${ }^{1}$ Centro Austral de Investigaciones Científicas (CADIC) - CONICET. ${ }^{2}$ Departamento de Ecología, Genética y Evolución \\ - IEGEBA (UBA-CONICET), Facultad de Ciencias Exactas y Naturales, Universidad de Buenos Aires. ${ }^{3}$ Departamento de \\ Biodiversidad y Biología Experimental, Facultad de Ciencias Exactas y Naturales, Universidad de Buenos Aires.
}

\begin{abstract}
Resumen. Los lagos urbanos son ecosistemas que tienden a una rápida eutrofización y que pueden desarrollar floraciones fitoplanctónicas en el período estival. En tres lagos urbanos de la Ciudad Autónoma de Buenos Aires (Regatas, Rosedal y Centenario), sometidos a diferentes estrategias de manejo, durante el período cálido septiembre 2014 - abril 2015 se estudió la estructura y la dinámica del fitoplancton, y se analizó la composición de especies y de grupos funcionales, y su relación con parámetros ambientales y variables físicas y químicas del agua. Los muestreos se llevaron a cabo quincenalmente. Los tres lagos difieren en sus características limnológicas, en las especies dominantes y en los grupos funcionales, así como en la biomasa de fitoplancton y la tendencia al desarrollo de floraciones. El lago del Rosedal (hipereutrófico) mostró una clara dominancia de cianobacterias potencialmente tóxicas que alcanzaron niveles de abundancia propios de las floraciones. El lago de Regatas presentó la mejor calidad del agua, con la mayor transparencia y la menor biomasa de fitoplancton. El lago Centenario, con la mayor conductividad y nitrógeno total, estuvo claramente dominado por diatomeas. Este trabajo evidencia que el análisis del fitoplancton a partir de grupos funcionales constituye una herramienta valiosa para monitorear lagos eutróficos urbanos y para aplicar estrategias adecuadas de manejo en los mismos.
\end{abstract}

[Palabras clave: lagos urbanos, eutrofización, floraciones algales, grupos funcionales]

\begin{abstract}
Aвstract. Phytoplankton dynamics in three urban lakes with different management strategies: analysis of the summer algal blooms. Urban lakes are ecosystems that tend to a rapid eutrophication, leading to the development of phytoplankton blooms in spring and summer. During the warm period, from September 2014 to April 2015 the structure and dynamics of the phytoplankton were studied in three urban lakes of the Buenos Aires city subjected to different strategies of management (Regatas, Rosedal, Centenario). The species composition and functional groups were analyzed, as well as the relationship between the community structure and the physical and chemical variables of the water and the main environmental parameters. Samplings were carried out fortnightly. The three lakes differ in their limnological characteristics, in the dominant species, their functional groups, as well as in the phytoplankton biomass, and their tendency to develop algal blooms. Rosedal Lake (hypereutrophic) showed a clear dominance of potentially toxic cyanobacteria that reached abundances proper of algal blooms. Regatas Lake presented the best water quality, with greater transparency and the lower phytoplankton biomass. Centenario Lake, with the highest values of conductivity and total nitrogen, was clearly dominated by diatoms. In this study it was evidenced that the analysis of the phytoplankton from functional groups constitutes a valuable tool for the monitoring of eutrophic urban lakes and to apply adequate management strategies in them.
\end{abstract}

[Keywords: urban lakes, eutrophication, algal blooms, functional groups]

\section{INTRODUCCIÓN}

Los lagos urbanos, también llamados "estanques" de acuerdo con la nomenclatura de Ringuelet (1962), son ecosistemas acuáticos artificiales que desde la antigüedad se construyen en las ciudades, principalmente con fines estéticos y recreativos (e.g., remo, pesca). Suelen ser pequeños y de escasa profundidad, proveen servicios ecosistémicos clave, ya que además de utilizarse para actividades recreativas contribuyen a regular el microclima en las ciudades (Schuler and Simpson 2001; Naselli-Flores 2008). Por lo general se encuentran emplazados en los parques, son nodos de biodiversidad en el

Editora asociada: Mariana Meerhoff 两 iri@ege.fcen.uba.ar ecosistema urbano (Garay and Fernández 2013) y aportan sus propias especies acuáticas. Por ser de pequeño tamaño y estar ubicados en ambientes con una actividad antrópica intensa son ecosistemas que tienden a eutrofizarse rápidamente $\mathrm{y}$, por ende, a mostrar un aumento de la biomasa de fitoplancton, con desarrollo de floraciones de algunas especies, principalmente del grupo de las cianobacterias (Waajen et al. 2014).

La eutrofización en lagos poco profundos fue asociada con la pérdida del estado de aguas claras (Moss 2007). Para este tipo de ecosistemas se describieron dos estados posibles de equilibrio alternativos (Scheffer et

Recibido: 11 de Abril de 2018

Aceptado: 3 de Diciembre de 2018 
al. 1993): un estado de aguas claras dominado por vegetación acuática sumergida a bajas concentraciones de nutrientes y un estado turbio con una alta biomasa algal a elevadas concentraciones denutrientes. Los mecanismos ecológicos involucrados son muchos, pero el centro del sistema de retroalimentación gira alrededor de la interacción entre la vegetación sumergida y la turbidez. Los lagos con una alta cobertura de macrófitas sumergidas tienden a tener una transparencia superior a la de los lagos con un rango de nutrientes similar en donde la vegetación es dispersa o está ausente.

Como consecuencia de la eutrofización de los lagos, las floraciones de algunas especies de fitoplancton son cada vez más frecuentes en todo el mundo (Wang et al. 2012), y constituyen una amenaza para la integridad ecológica y la sostenibilidad de los cuerpos de agua. En algunos ecosistemas, las floraciones tienen la cualidad de mostrar una clara temporalidad condicionada por los factores ambientales (Murrel and Lores 2004). En aguas eutróficas, el cambio climático puede ser un catalizador potencial en la expansión de las floraciones de cianobacterias a escala global, con lo que se beneficia el incremento de las tasas de crecimiento, persistencia, actividad y distribución geográfica (Paerl and Huisman 2009). En general, suelen ocurrir en verano, pero es probable que, como consecuencia del cambio climático, persistan durante el otoño en numerosos cuerpos de agua, tal como se ha visto en los últimos años (e.g., Izaguirre et al. 2015).

Debido a la tendencia a eutrofizarse, uno de las principales preocupaciones vinculadas a los lagos urbanos es mantener la calidad del agua. En este contexto, el análisis de los niveles de nitrógeno y fósforo total resulta imprescindible para evaluar el estado trófico de un cuerpo de agua (Hellström et al. 2000). No obstante, en la legislación europea se realizó el primer intento en el tránsito de los estándares de calidad físicos y químicos a estándares ecológicos, con el objeto de establecer y conciliar límites de estados ecológicos. Para lograrlo se parte de la definición de qué es un lago en buen estado. Poikane et al. (2014) lo definen como aquel que tenga baja probabilidad de floraciones de cianobacterias y una baja probabilidad en cuanto a la disminución significativa de las macrófitas colonizadoras del fondo. Asimismo, estos autores determinaron los límites entre un estado bueno y moderado a partir de la respuesta entre la abundancia del fitoplancton y los efectos ecológicos de la eutrofización, utilizando la clorofila a como la variable de análisis de los cambios ecológicos.

El análisis de la composición y la biomasa de la comunidad fitoplanctónica es clave en un contexto de gestión y monitoreo ambiental (Guo et al. 2010). Los cambios en la composición de especies del fitoplancton son el resultado de la interacción entre las propiedades físicas, químicas o biológicas de un ambiente acuático, y de la capacidad de respuesta de las especies (Reynolds 1986, 1997, y citas en estas fuentes; Dignum et al. 2005). Con el fin de arribar a un mayor entendimiento del funcionamiento de los ecosistemas, desde hace tiempo los ecólogos intentan agrupar organismos con características estructurales y funcionales similares (Salmaso and Padisák 2007). Las primeras aproximaciones a los grupos funcionales fitoplanctónicos surgieron a partir de los conceptos de fitosociología, en la cual las asociaciones se definieron como grupos de especies que suelen encontrarse típicamente juntas (e.g., Margalef 1978; Reynolds 1980,1984), y en la que las especies de una asociación particular comparten atributos ecológicos similares. En particular, una de las clasificaciones más usadas y revisadas es la de Reynolds et al. (2002), quienes propusieron una serie de grupos funcionales de fitoplancton de agua dulce (codones) sobre la base de aspectos filogenéticos, rasgos morfológicos y frecuencia de aparición en distintos tipos de ambientes. Recientemente, Padisák et al. (2009) actualizaron esta clasificación. Por otro lado, Kruk et al. (2010) propusieron una clasificación más simple basada en aspectos morfológicos objetivos. Estas clasificaciones funcionales fueron implementadas con anterioridad en distintos tipos de ambientes acuáticos (Izaguirre et al. 2012, 2015; Saad et al. 2013; Crossetti et al. 2013; Gillett et al. 2015) y constituyen herramientas excelentes para programas de monitoreo, ya que facilitan la toma de decisiones por parte de los gestores de la calidad del agua (e.g., Kruk et al. 2010; Saad and El-Karim 2015).

La Ciudad Autónoma de Buenos Aires (CABA), ubicada sobre la margen sur del Río de la Plata, cuenta con 14 cuerpos de agua artificiales. Al ser una ciudad costera, sobrecargada poblacionalmente, se enfrenta al deterioro de sus ecosistemas (Timmerman and White 1997). En los lagos urbanos de Buenos Aires se llevaron a cabo algunas investigaciones centradas en el fitoplancton 
con el objeto de analizar la calidad del agua y el desarrollo de floraciones algales. Uno de los estudios pioneros en este sentido fue el de Izaguirre et al. (1986) para los lagos del Jardín Japonés y del Rosedal, en el que se reportaron floraciones estivales masivas para ambos cuerpos de agua. Luego, el trabajo de Ehrenhaus and Vigna (2006) se centró en el análisis de un evento de floración masiva de Microcystis aeruginosa en el lago del Planetario, que desencadenó la muerte de peces y aves. Otros estudios previos en CABA se focalizaron en cuerpos de agua de la Reserva Ecológica Costanera Sur. En particular, en la laguna Los Coipos, Fazio and O'Farrell (2005) evaluaron la composición del fitoplancton antes, durante y después de que sufriera una salinización secundaria producto de acciones de manejo incorrectas por el llenado con agua de un acuífero con mayor conductividad. En el mismo cuerpo de agua, Avigliano et al. (2014) valoraron los cambios de la estructura del fitoplancton partiendo de un estado de sequía a uno de llenado. Por otro lado, en el trabajo de Sinistro et al. (2013) se analizó la estructura del fitoplancton y las fluctuaciones de variables limnológicas en las lagunas de Los Patos y de Las Gaviotas de la misma reserva durante un ciclo hidrológico completo, y a partir de sus resultados los autores realizaron recomendaciones para el manejo y restauración de estos cuerpos de agua.

En este trabajo se evaluó el estado trófico y la sucesión temporal del fitoplancton, y su relación con los factores físicos y químicos en tres lagos urbanos de CABA durante el período cálido 2014-2015. La comunidad fitoplanctónica fue analizada a nivel de especies y de grupos funcionales, y se usaron las dos clasificaciones funcionales antes mencionadas (Reynolds et al. 2002; Kruk et al. 2010). En particular, se analizó el potencial desarrollo de floraciones nocivas durante el período estival. La selección de los tres lagos se basó en observaciones previas que revelaron ensambles fitoplanctónicos diferentes y en el conocimiento de que los tres están sometidos a diferentes tipos de manejo.

A partir de este conocimiento, y teniendo en cuenta que las estrategias de manejo afectan las variables limnológicas de los lagos, como hipótesis de trabajo se plantea que las características limnológicas de los lagos urbanos condicionan la estructura del fitoplancton $y$, particularmente, la composición de los grupos funcionales, lo que determina diferencias entre cuerpos de agua de una misma ciudad. Se espera que: a) altos períodos de residencia del agua, acompañados por la ausencia de macrófitas sumergidas, promuevan el deterioro de la calidad del agua, la formación de floraciones (principalmente cianobacterias) y la dominancia de grupos funcionales asociados a cuerpos de agua ricos en nutrientes, b) sistemas con flujo y recirculado del agua faciliten el establecimiento de grupos funcionales tolerantes a la mezcla, y prevengan el desarrollo de floraciones, y c) los grupos funcionales del fitoplancton permitan una caracterización adecuada de las comunidades en los lagos urbanos, y que se constituyan en una herramienta útil para su monitoreo con el fin de implementar estrategias de manejo que faciliten su conservación.

\section{Materiales y Métodos}

\section{Ambientes estudiados}

Se estudiaron tres lagos urbanos (Regatas, Rosedal y Centenario) ubicados en parques de CABA (Figura 1). Esta ciudad es una gran urbe
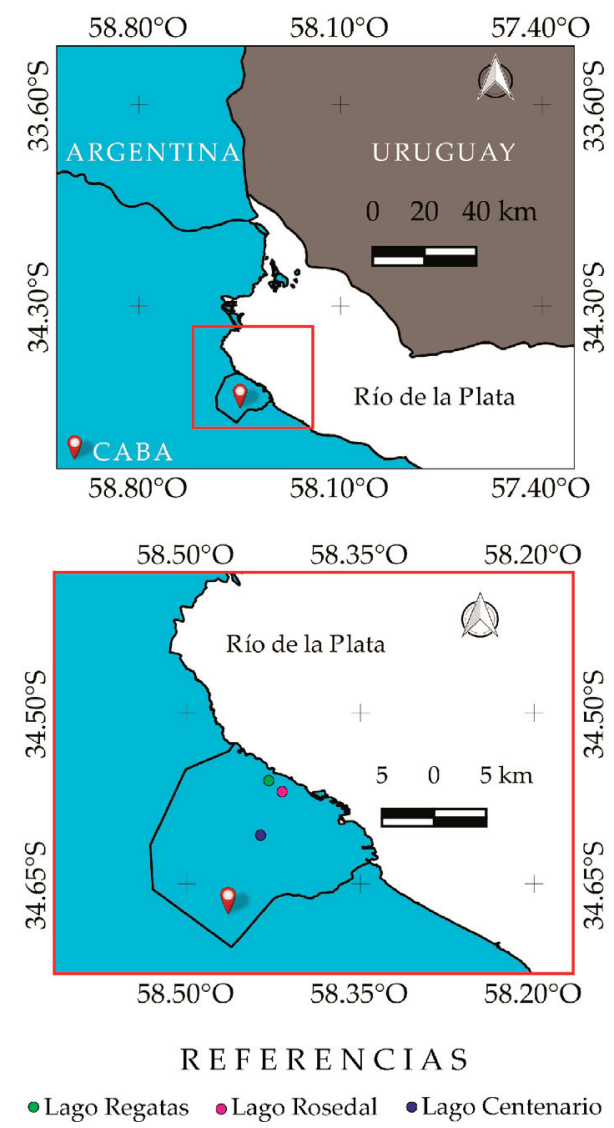

Figura 1. Localización de los tres lagos urbanos estudiados en la Ciudad Autónoma de Buenos Aires.

Figure 1. Geographic position of the three studied urban lakes in Buenos Aires City. 
de 2.9 millones de habitantes, de acuerdo con el censo de 2010. Está localizada en la Región Pampeana (Argentina), que se caracteriza por ser una planicie con pendiente escasa donde se registran eventos hidrológicos extremos de diversa severidad (Gómez and Toresani 1998; Scarpati and Capriolo 2013).

Los tres lagos presentan poca profundidad (prof. máxima $<4 \mathrm{~m}$ y media entre 1.5 y $2 \mathrm{~m}$ ) y su finalidad es principalmente ornamental y recreativa. Un gran número de personas los visita todos los días, y en particular los fines de semana. Los tres cuerpos de agua difieren en su forma de manejo, llevado a cabo por el Gobierno de la ciudad. En la actualidad, el lago de Rosedal no cuenta con un sistema de recirculado de agua que funcione correctamente, y es abastecido sobre todo por agua de lluvia. El lago del Parque Centenario tiene un ingreso de agua subterráneo y dispone de una serie de filtros. El lago de Regatas tiene un sistema de recirculado de agua en correcto funcionamiento, con entrada desde los tanques de saneamiento de la empresa AySA (Agua y Saneamiento Argentinos S.A.), y es el único que poseía macrófitas sumergidas en el período de muestreo. Recientemente (año 2018), este lago fue reacondicionado por completo para ser usado durante los Juegos Olímpicos de la Juventud. En la Tabla 1 se resumen las principales características morfométricas de los tres lagos, sus rasgos principales y formas de manejo.

\section{Condiciones meteorológicas}

Los datos meteorológicos para el período de estudio se obtuvieron de las estaciones de Caballito (más cercana al lago Centenario) y de Ciudad Universitaria (más cercana a los lagos Rosedal y Regatas) (Figura 2). Aunque los lagos se encuentran en la misma ciudad, cabe señalar que la temperatura media fue superior en la estación de Caballito debido a un efecto de isla de calor urbana con respecto a la obtenida en la estación de Ciudad Universitaria. Enero y febrero fueron los meses más cálidos, con temperaturas medias de $\sim 24{ }^{\circ} \mathrm{C}$ para la estación de Ciudad Universitaria y de $25{ }^{\circ} \mathrm{C}$ para la estación de Caballito. En cuanto a la precipitación acumulada, el mes con mayor precipitación para la estación de Caballito fue octubre de 2014, y para la de Ciudad Universitaria, noviembre del mismo año. Considerando todo el período de muestreo se registró una mayor precipitación en la estación de la Ciudad Universitaria $(1148.4 \mathrm{~mm}$ ) que en la estación de Caballito $(783 \mathrm{~mm})$. Hacia fines de octubre de 2014, en la zona centro y norte del país ocurrió una ola de calor; en CABA se registró una temperatura máxima de 35.6 ${ }^{\circ} \mathrm{C}$ (27 de octubre, Observatorio Central de Buenos Aires), un valor que superó la máxima

Tabla 1. Principales características de los lagos urbanos estudiados en la Ciudad Autónoma de Buenos Aires, Argentina.

Table 1. Main characteristics of the urban lakes studied in Buenos Aires City, Argentina.

\begin{tabular}{|c|c|c|c|c|}
\hline Lago urbano & Parque & Perímetro & Superficie & Forma de manejo durante el período de estudio \\
\hline Centenario & Centenario & $\sim 485 \mathrm{~m}$ & $\sim 7390 \mathrm{~m}^{2}$ & $\begin{array}{l}\text { Recirculado del agua y disposición de filtros. Ingreso de } \\
\text { agua subterránea (Gobierno de CABA, comunicación } \\
\text { personal, enero de 2015). Carece de macrófitas } \\
\text { sumergidas. }\end{array}$ \\
\hline Regatas & $\begin{array}{l}\text { Tres de } \\
\text { Febrero }\end{array}$ & $1836 \mathrm{~m}$ & $98226 \mathrm{~m}^{2}$ & $\begin{array}{l}\text { Posee entrada de agua desde los tanques de tratamiento } \\
\text { de la empresa Agua y Saneamiento Argentinos S.A. } \\
\text { (AySA) y salida de agua al Río de la Plata (Gobierno de } \\
\text { CABA, comunicación personal, octubre de 2016). En el } \\
\text { período de muestreo el entorno del lago Regatas fue } \\
\text { sometido a mejoras (abril-noviembre de 2014), entre las } \\
\text { que se incluyó la mejora del sistema de drenaje mediante } \\
\text { un badén y una lomada perimetral que canaliza las } \\
\text { aguas de lluvia hacia el lago (Buenos Aires Ciudad } \\
\text { 2014). Durante el período de muestreo contaba con un } \\
\text { desarrollo moderado de macrófitas sumergidas. }\end{array}$ \\
\hline Rosedal & $\begin{array}{l}\text { Tres de } \\
\text { Febrero }\end{array}$ & $\sim 1655 \mathrm{~m}$ & $\sim 45772 \mathrm{~m}^{2}$ & $\begin{array}{l}\text { En la actualidad el lago tiene aguas estancadas y capta } \\
\text { agua de lluvia. No obstante, hace } 7 \text { años contaba con } \\
\text { un sistema de flujo que incluía } 8 \text { bombas, hoy averiadas } \\
\text { (Gobierno de CABA, comunicación personal, octubre de } \\
\text { 2016). Carece de macrófitas sumergidas. }\end{array}$ \\
\hline
\end{tabular}




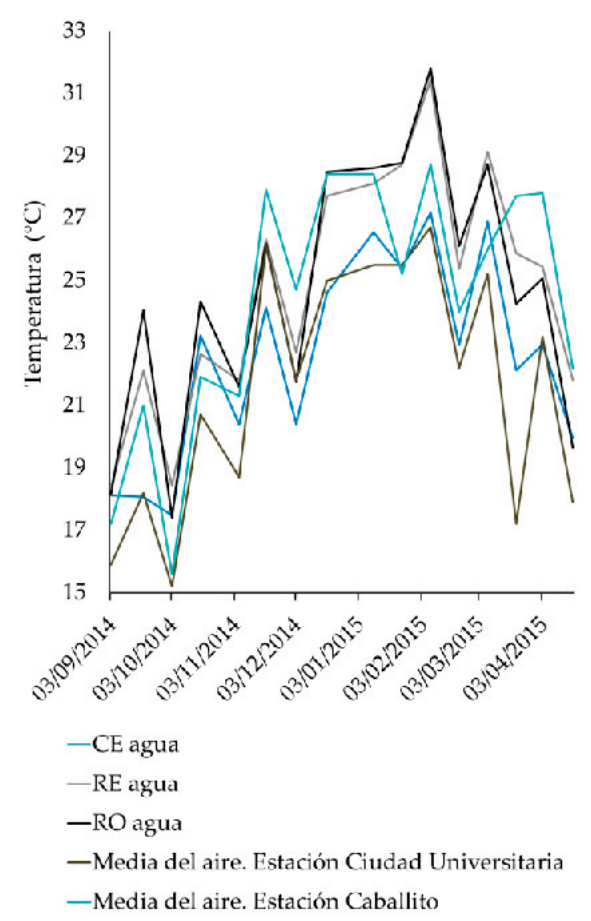

Figura 2. Variación de la temperatura del agua en los lagos urbanos (CE: Centenario, RE: Regatas, RO: Rosedal), y del aire en las estaciones meteorológicas. CABA, Argentina.

Figure 2. Water temperature variation in urban lakes (CE: Centenario, RE: Regattas, RO: Rosedal), and of the air in the meteorological stations. CABA, Argentina.

observada de todos los octubres en el período 2001-2010 (Pittaluga 2014).

\section{Diseño de muestreo}

Entre septiembre de 2014 y abril de 2015 se realizaron 8 muestreos quincenales, con los que se abarcó todo el período cálido (primavera y verano). El objetivo fue evaluar, con una alta frecuencia, las variaciones en el fitoplancton durante la época de mayor crecimiento algal $\mathrm{y}$, es especial, cuando se producen las floraciones.

En cada cuerpo de agua se recolectaron muestras sub-superficiales para llevar a cabo análisis químicos de clorofila $a$ y para análisis cualitativos y cuantitativos de fitoplancton. Además, se midieron in situ las siguientes variables limnológicas: oxígeno disuelto (con un sensor de campo HANNA modelo HI 9146), conductividad (con un sensor ALTRONIX modelo CT), temperatura y $\mathrm{pH}$ (con un sensor HANNA modelo HI 991301) y transparencia (con la profundidad de disco de Secchi).

Las muestras de fitoplancton para análisis cualitativos se obtuvieron con una red de
$18 \mu \mathrm{m}$ de poro y se fijaron con formol al $4 \%$. Para el análisis cuantitativo del fitoplancton se obtuvieron muestras sin filtrar y se fijaron con lugol acidificado al 1\%. Los recuentos de fitoplancton se llevaron a cabo en cámaras de sedimentación de volumen adecuado de acuerdo a la densidad algal; se siguió la metodología descripta por Utermöhl (1958) y se usó un microscopio invertido Olympus CKX41. El error de los recuentos se estimó de acuerdo con Venrick (1978). La identificación de las especies se llevó a cabo con un microscopio binocular (Olympus CX31); las especies fueron fotografiadas con un software incorporado al microscopio y se consultó la bibliografía adecuada para cada grupo. Las especies de fitoplancton se clasificaron por medio de dos clasificaciones funcionales: la de Reynolds et al. (2002), actualizada por Padisák et al. (2009), y la de Kruk et al. (2010).

Se calculó el biovolumen de cada especie algal en base a mediciones realizadas al microscopio a 1000X. Las algas se asemejaron a cuerpos geométricos de acuerdo con el procedimiento descripto por Hillebrand et al. (1999).

Para estimar la concentración de clorofila $a$ se filtraron las muestras de agua a través de filtros de fibra de vidrio Whatman GF/F. Los filtros se guardaron en freezer $\left(-20^{\circ} \mathrm{C}\right)$ hasta la extracción de los pigmentos. Se utilizó acetona como solvente de extracción. Las estimaciones de clorofila se llevaron a cabo espectrofotométricamente antes y después de acidificar con $\mathrm{HCl}$ (clorofila libre de feopigmentos), se midieron las absorbancias a 665 y $750 \mathrm{~nm}$ y se calcularon las concentraciones con las ecuaciones propuestas por Marker et al. (1980).

Para el análisis de nutrientes totales (NT y PT) se sometieron muestras de agua sin filtrar a una digestión alcalina previa (Koroleff 1983). La determinación de nitrógeno y fósforo total se realizó por métodos espectrofotométricos (espectrofotómetro Hach y sus correspondientes kits de reactivos) y se aplicaron técnicas estándares (APHA 2005).

\section{Análisis de datos}

Para analizar la existencia de diferencias significativas entre los lagos con respecto a las variables limnológicas para el período estudiado se llevaron a cabo modelos de regresión de mínimoscuadrados generalizados con medidas repetidas para cada una de las variables, considerando la interacción entre el 
lago y mes muestreado, y se seleccionaron los modelos que mejor ajuste tenían de acuerdo con el coeficiente de Akaike (AIC). Luego, se realizaron comparaciones post-hoc de Tukey para los modelos sin interacción y de Tukey ajustado para los modelos con interacción. Se consideró Ho: las medias de los grupos no son significativamente diferentes, y Ha: las medias de los grupos son significativamente diferentes. Para el contraste de hipótesis, el alfa global fue 0.05 y se usó el método de Bonferroni secuencial para determinar el alfa individual. Estos análisis se realizaron por medio del software R (R-Core team 2016).

Para apreciar la variación estival de las clases algales en los diferentes lagos, a partir de datos transformados mediante la función de raíz cuadrada se construyeron diagramas kite (usando $\operatorname{Excel}^{\circledR}$ ) de biovolumen (biov.) y densidad (dens.). Con el objeto de jerarquizar la dominancia de especies se construyeron diagramas Olmstead-Tukey a partir de la densidad. También se elaboraron diagramas kite en base al biovolumen de los grupos funcionales según la clasificación de Reynolds et al. (2002), actualizada por Padisák et al. (2009), y de Kruk et al. (2010).

Por otro lado, se llevó a cabo un análisis de correspondencia canónica (CCA) para evaluar la varianza explicada por las variables limnológicas del: a) biovolumen de especies, b) biovolumen de los grupos funcionales propuestos por Reynolds et al. (2002), y c) un análisis de redundancia (RDA) de los grupos funcionales basados en la morfología (Kruk et al. 2010). Previamente, se llevó a cabo un análisis de correspondencias sin tendencia (DCA) para seleccionar un modelo lineal o unimodal de la curva de respuesta del biovolumen según el largo del gradiente ambiental. A la vez, se efectuó la prueba de permutación de Monte Carlo para analizar la significancia del análisis. Se utilizó la selección forward para evaluar qué variables resultaban significativas. Cabe señalar que se quitó peso a las especies y grupos funcionales raros y se aplicó transformación logarítmica. El análisis se realizó mediante el uso del paquete estadístico CANOCO 4.5 (ter Braak and Smilauer 2002).

\section{Resultados}

\section{Características limnológicas}

Los valores medios de $\mathrm{pH}$ variaron en los tres lagos urbanos entre 6.92 y 9.13, y en general fueron mayores en Rosedal, intermedios en Centenario y más bajos en Regatas. La conductividad fue mucho más alta en

Tabla 2. Variables limnológicas (valores medios, mínimos, máximos y desviación estándar). Estanques urbanos (CE: Centenario, RE: Regatas, RO: Rosedal), de septiembre de 2014 a abril de 2015. Buenos Aires, Argentina.

Table 2. Limnological variables (average, minimum, maximum values and standard deviation). Urban ponds (CE: Centenario, RE: Regatas, RO: Rosedal) from September 2014 to April 2015. Buenos Aires, Argentina.

\begin{tabular}{lccc}
\hline & $\mathrm{CE}$ & $\mathrm{RE}$ & $\mathrm{RO}$ \\
\hline Temperatura $\left({ }^{\circ} \mathrm{C}\right)$ & 22.52 & 24.75 & 24.68 \\
& $(17.47-27.17)$ & $(18.4-31.43)$ & $(17.4-31.8)$ \\
Oxígeno disuelto $(\mathrm{mg} / \mathrm{L})$ & \pm 3.2 & \pm 3.8 & \pm 4.14 \\
& 10.56 & 6.37 & 9.38 \\
& $(6.92-26.34)$ & $(2.31-10.4)$ & $(3.82-18.73)$ \\
$\mathrm{pH}$ & \pm 5.26 & \pm 2.77 & \pm 4.1 \\
& 8.12 & 6.92 & 9.13 \\
& $(6.5-8.67)$ & $(6-7.82)$ & $(7.37-9.81)$ \\
Conductividad $(\mu \mathrm{S} / \mathrm{cm})$ & \pm 0.6 & \pm 0.58 & \pm 0.72 \\
& 1436.8 & 253.8 & 867 \\
$\mathrm{NT}(\mathrm{mg} / \mathrm{L})$ & $(831-3320)$ & $(179-367.2)$ & $(294-1591.2)$ \\
& \pm 731 & \pm 56.6 & \pm 405.3 \\
PT $(\mathrm{mg} / \mathrm{L})$ & 9.93 & 1.37 & 1.97 \\
& $(7.48-12.17)$ & $(1.01-1.9)$ & $(0.67-3.46)$ \\
& \pm 1.51 & \pm 0.25 & \pm 0.76 \\
Clorofila $a(\mu \mathrm{g} / \mathrm{L})$ & 0.19 & 0.14 & 0.40 \\
& $(0.09-0.44)$ & $(0.04-0.32)$ & $(0.17-0.79)$ \\
& \pm 0.11 & \pm 0.08 & \pm 0.19 \\
& 19.29 & 7.38 & 91.22 \\
& $(2.51-48.03)$ & $(2.04-16.30)$ & $(24.45-173.17)$ \\
\end{tabular}


Centenario, con dos valores extremos (3320 $\mu \mathrm{S} / \mathrm{cm}$ el 18 de diciembre de 2014 y 2861.9 $\mu \mathrm{S} / \mathrm{cm}$ el 21 de febrero de 2015), intermedia en Rosedal y menor en Regatas. El oxígeno disuelto varió entre 6.37 y $10.56 \mathrm{mg} / \mathrm{L}$; los valores fueron mayores en Centenario, intermedios en Rosedal y menores en Regatas. La temperatura del agua siguió un mismo patrón en los tres lagos, lo que refleja los cambios ambientales, con valores máximos en febrero (Tabla 2, Figura 2).

En el lago Centenario se registraron valores intermedios de clorofila $a$, con dos picos, uno el 18 de noviembre de $2014(48.03 \mu \mathrm{g} / \mathrm{L})$ y el otro el 7 de marzo de 2015 (37.08 $\mu \mathrm{g} / \mathrm{L})$. En Regatas se observaron los valores mínimos, que no superaron nunca los $16.30 \mu \mathrm{g} / \mathrm{L}$. En Rosedal, los valores fueron notoriamente mayores, con concentraciones típicas de lagos hipereutróficos (picos >100 $\mu \mathrm{g} / \mathrm{L}$ ), y descensos marcados después de lluvias intensas. En particular, en el muestreo del 5 de noviembre de 2014, luego de un período de intensas lluvias (238.4 mm acumulados entre el 28 de octubre y 4 de noviembre), la clorofila $a$ fue de $29.88 \mu \mathrm{g} / \mathrm{L}$ y el valor del nitrógeno total fue el mínimo registrado $(0.67 \mathrm{mg} / \mathrm{L})$.

De acuerdo con la profundidad del disco de Secchi, el lago de Regatas fue el más transparente, con lecturas que siempre llegaron al fondo. Por el contrario, en el lago del Rosedal siempre se registraron lecturas muy bajas que oscilaron entre 13.5 y $33 \mathrm{~cm}$.
En el lago Centenario, en la mayoría de los muestreos se observó una profundidad del disco de Secchi que llegó hasta el fondo; en otras fechas varió entre 42 y $57 \mathrm{~cm}$.

De acuerdo con los rangos de estado trófico propuestos por OCDE (1982) y el índice de Carlson (1977), utilizando la clorofila a como estimador, los valores obtenidos en el período de muestreo permiten clasificar al Rosedal como un cuerpo de agua hipereutrófico (promedio del índice del estado trófico TSI

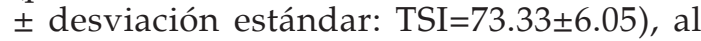
Centenario como eutrófico (TSI=57.45 \pm 7.36 ), y al Regatas como mesotrófico (TSI $=48.69 \pm 5.84$ ). Sin embargo, si tenemos en cuenta al fósforo total, de acuerdo con el índice de Carlson, los tres lagos serían hipereutróficos (Rosedal: TSI=89.17 \pm 6.73 ; Centenario: $\mathrm{TSI}=78.23 \pm 7.55$; Regatas: TSI=73.38 \pm 7.83 ).

Las comparaciones de Tukey realizadas en relación a la temperatura del agua y el nitrógeno total mostraron que el lago Centenario presentó características que lo diferencian de los lagos Regatas y Rosedal. Se observaron diferencias significativas en el $\mathrm{pH}$, la conductividad, el fósforo total y la clorofila a para todas las comparaciones de lagos realizadas (Tabla 3 ).

Entre Rosedal y Centenario no se encontraron diferencias significativas en cuanto a la concentración de oxígeno disuelto. Entre Regatas y Centenario no se hallaron diferencias

Tabla 3. Pruebas de medidas repetidas de las variables medidas in situ (CE: Centenario, RE: Regatas, RO: Rosedal), de septiembre de 2014 a abril de 2015. Profundidad de disco de Secchi (variable cualitativa ordinal en cm: 1) 13.5-21.5; 2) 21.6-29.6; 3) 29.7-37.7; 4) 37.8-45.8; 5) 45.9-53.9; 6) 54-62; 7) fondo]. $\alpha$-global=0.05; $\alpha$-individual=Bonferroni secuencial. Ho: las medias no son significativamente diferentes $(P>\alpha)$. Ha: las medias son significativamente diferentes*.

Table 3. Tests of repeated measures of the variables measured in situ (CE: Centenario, RE: Regatas, RO: Rosedal), from September 2014 to April 2015. Secchi depth [qualitative ordinal variable in cm: 1) 13.5-21.5; 2) 21.6-29.6;3) 29.7-37.7; 4) $37.8-45.8$; 5) 45.9-53.9; 6) 54-62;7) background]. $\alpha$-global=0.05; $\alpha$-individual=Bonferroni sequential. Ho: means are not significantly different $(P>\alpha)$. Ha: means are significantly different *

\begin{tabular}{|c|c|c|c|c|c|c|}
\hline Variable & $\mathrm{R}^{2}$ ajustado & $\begin{array}{c}\text { Interacción, } \\
\text { lago:mes, } \\
P \text {-valor }\end{array}$ & $\begin{array}{c}\text { ANOVA MR, } \\
P \text {-valor efecto } \\
\text { principal (lago) }\end{array}$ & $\begin{array}{l}\text { RE-CE } \\
P \text {-valor }\end{array}$ & $\begin{array}{c}\text { mparaciones post } \\
\text { RE-RO } \\
P \text {-valor }\end{array}$ & $\begin{array}{l}\text { hoc-CE } \\
P \text {-valor }\end{array}$ \\
\hline $\mathrm{pH}$ & 0.81 & 0.56 & $<.0001$ & $<2 \mathrm{e}-16^{*}$ Tukey & $<2 \mathrm{e}-16^{*}$ Tukey & $<2 \mathrm{e}-16^{*}$ Tukey \\
\hline Conductividad & 0.69 & $<.0001$ & $<.0001$ & $\begin{array}{c}<.0001^{*} \text { Tukey } \\
\text { ajustado }\end{array}$ & $\begin{array}{c}<.0001^{*} \text { Tukey } \\
\text { ajustado }\end{array}$ & $\begin{array}{c}<.0001^{*} \text { Tukey } \\
\text { ajustado }\end{array}$ \\
\hline Oxígeno disuelto & 0.18 & 0.34 & 0.0026 & $0.103^{*}$ Tukey & $<0.001^{*}$ Tukey & 0.207 Tukey \\
\hline NT & 0.95 & 0.24 & $<.0001$ & $<0.001^{*}$ Tukey & 0.203 Tukey & $<0.001^{*}$ Tukey \\
\hline PT & 0.43 & 0.03 & $<.0001$ & 0.00977* Tukey & $<0.001^{*}$ Tukey & $<0.001^{*}$ Tukey \\
\hline Clorofila $a$ & 0.83 & 0.0003 & $<.0001$ & $\begin{array}{c}<.0001^{*} \text { Tukey } \\
\text { ajustado }\end{array}$ & $\begin{array}{c}<.0001^{*} \text { Tukey } \\
\text { ajustado }\end{array}$ & $\begin{array}{c}<.0001^{*} \text { Tukey } \\
\text { ajustado }\end{array}$ \\
\hline $\begin{array}{l}\text { Transparencia } \\
\text { Secchi }\end{array}$ & 0.92 & - & $<.0001$ & 0.127 Tukey & $<1 \mathrm{e}-04^{*}$ Tukey & $<1 \mathrm{e}-04^{*}$ Tukey \\
\hline Temp. del agua & 0.61 & 0.38 & $<.0001$ & $<1 \mathrm{e}-07^{*}$ Tukey & 0.999 Tukey & $<1 \mathrm{e}-07^{*}$ Tukey \\
\hline
\end{tabular}


en la transparencia del agua medida por la profundidad del disco de Secchi (Tabla 3).

\section{Fitoplancton}

Biovolumen y densidad de los grupos taxonómicos. Para todo el período demuestreo, el mayor biovolumen fitoplanctónico se registró en Rosedal $\left(27470.5 \times 10^{6} \mu^{3} /\right.$
$\mathrm{mL})$, seguido por Centenario $\left(2707.2 \times 10^{6}\right.$ $\left.\mu^{3} / \mathrm{mL}\right)$ y Regatas $\left(321.7 \times 10^{6} \mu \mathrm{m}^{3} / \mathrm{mL}\right)$. Bacillariophyceae fue el grupo que dominó en Centenario ( $68 \%$ biov., $40 \%$ dens.) y en Regatas (58\% biov., 33\% dens.), co-dominado en Centenario por Cyanobacteria (13\% biov., $26 \%$ dens.) y Chlorophyceae (9\% biov., $26 \%$ dens.). En Regatas, los grupos co-dominantes fueron Cryptophyceae ( $24 \%$ biov., $54 \%$ dens.)

a)
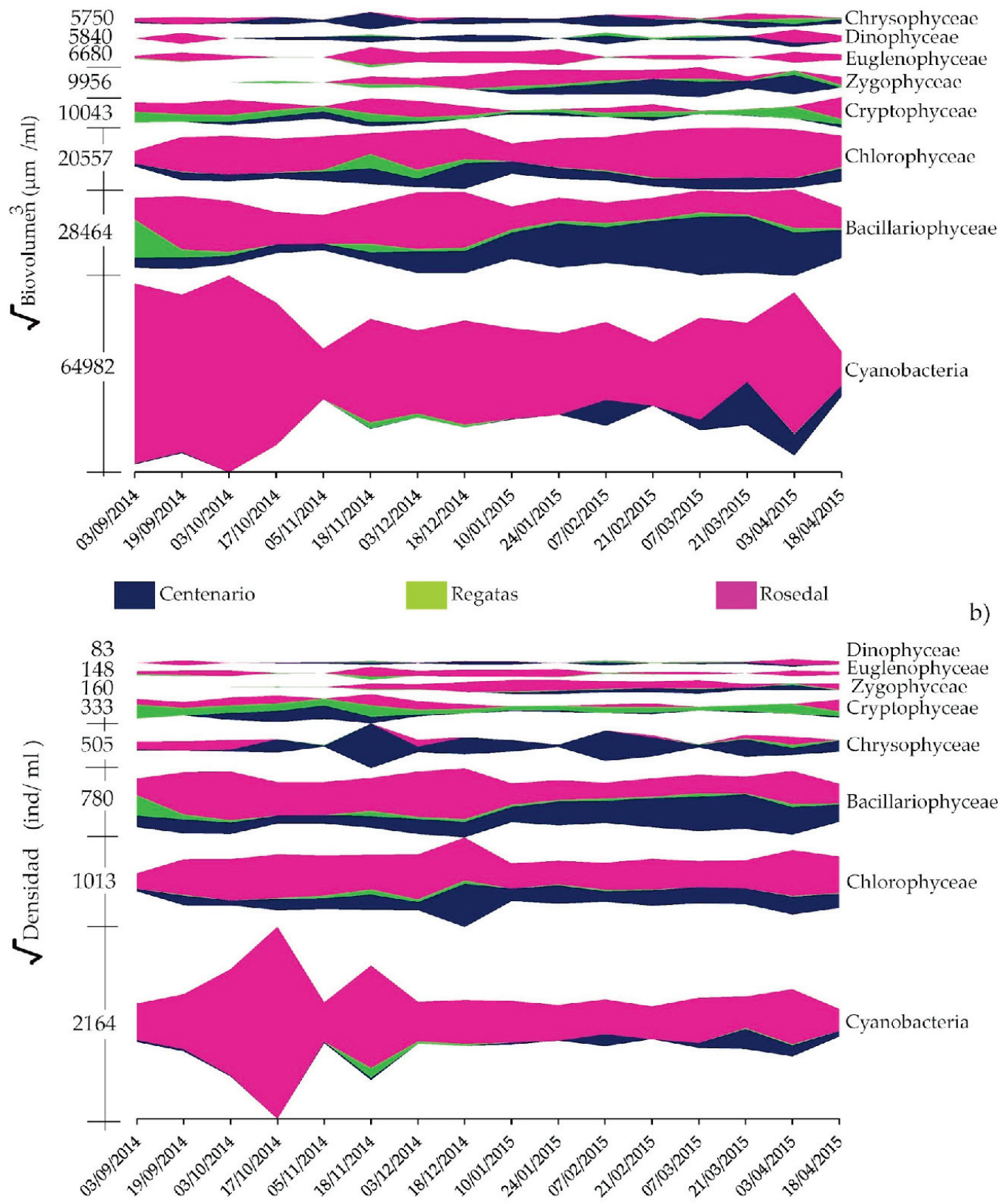

Figura 3. Diagramas kite de la variación estival de: a) el biovolumen y b) la densidad de los grupos taxonómicos del fitoplancton en los diferentes lagos urbanos.

Figure 3. Kite diagrams of the summer variation of a) biovolume and b) density of phytoplankton taxa in the different urban lakes. 
GF: Kruk et al. (2010)
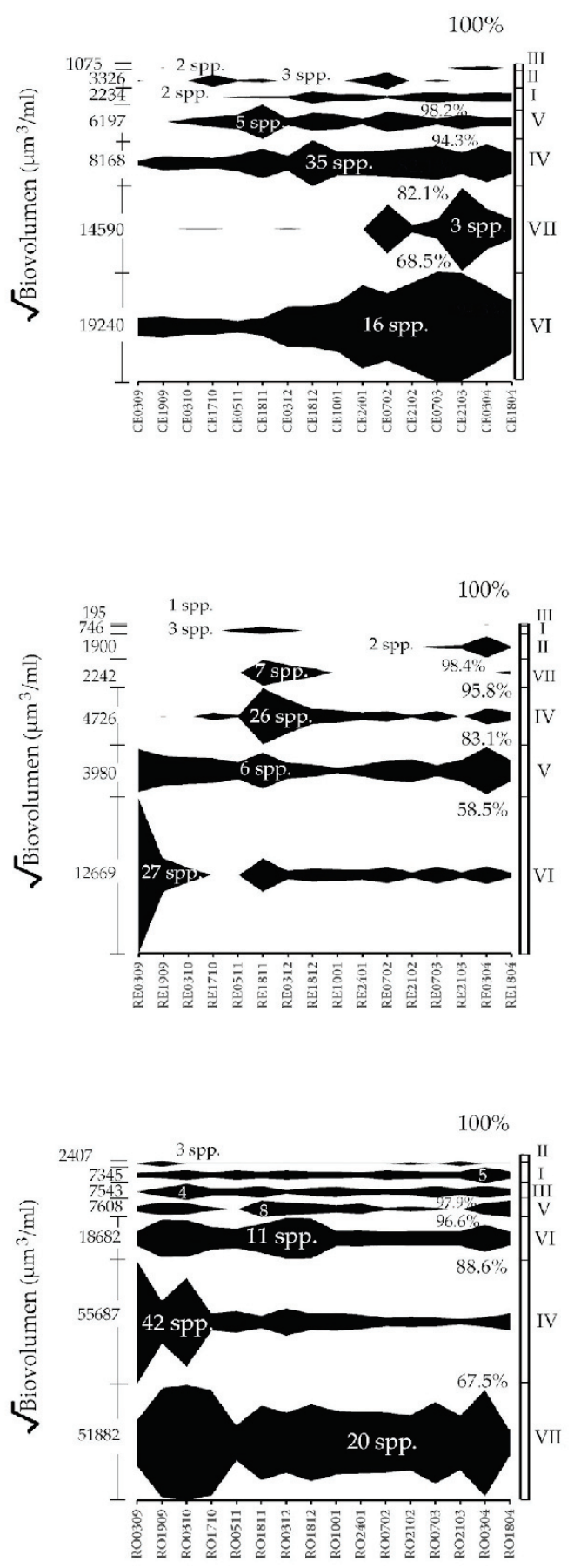

GF: Reynolds et al. (2002), actualizados por Pádisak et al. (2009)

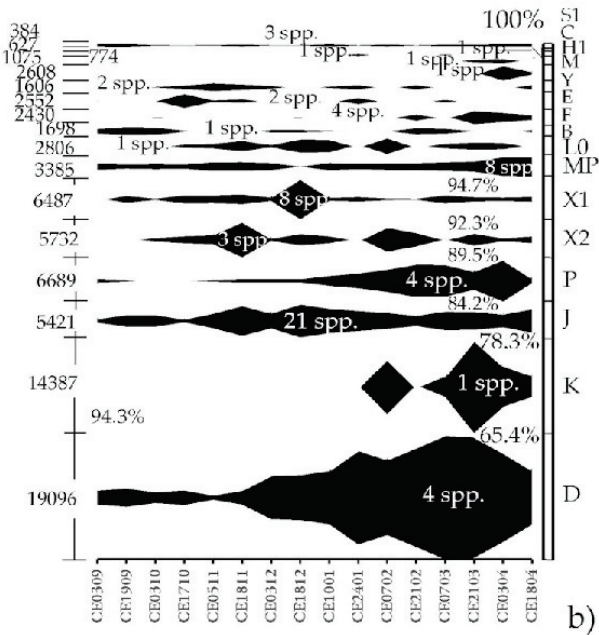

b)

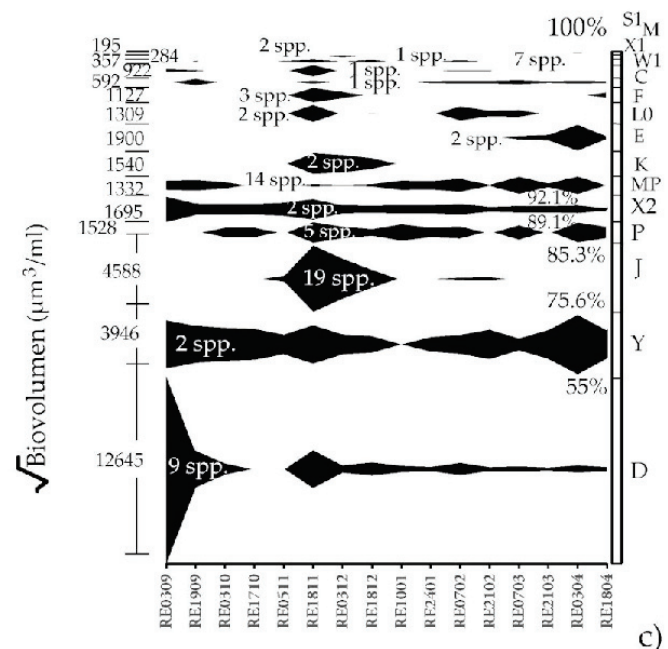

c)

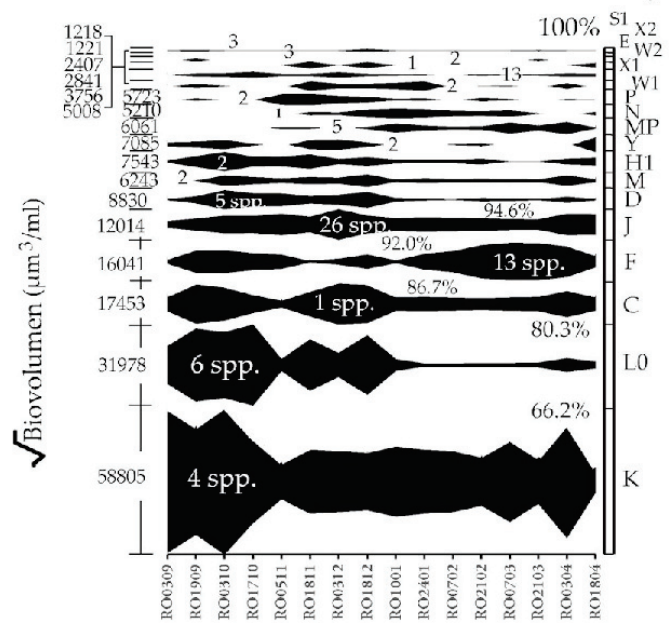

Figura 4. Diagramas kite del biovolumen de los grupos funcionales según Kruk y Reynolds, actualizados por Padisák, lagos: a) Centenario, b) Regatas y c) Rosedal, de septiembre de 2014 a abril de 2015. Buenos Aires, Argentina.

Figure 4. Kite diagrams of the biovolume of the functional groups according to Kruk and Reynolds updated by Padisák, lagos: a) Centenario, b) Regatas, and c) Rosedal, from September 2014 to April 2015 
y Chlorophyceae (11\% biov., $4 \%$ dens.). En Rosedal dominó Cyanobacteria (82\% biov., $66 \%$ dens.) y se observó co-dominancia de Chlorophyceae ( $8 \%$ biov., 18\% dens.) y Bacillariophyceae ( $8 \%$ biov., 14\% dens.) (Figura 3a y b).

En Rosedal, las cianobacterias estuvieron presentes durante todo el período de muestreo, y presentaron su mayor biovolumen en septiembre y en octubre de 2014 (Figura $3 a)$, lo que representa en ambos casos el $56.2 \%$ del biovolumen total. En septiembre, las especies que aportaron el $98.3 \%$ del biovolumen fueron Coelosphaerium aerugineum (55.3\%), Aphanocapsa incerta (18.5\%), Snowella lacustris (15.7\%) y Aphanocapsa spp. (8.8\%). En octubre constituyeron el $97 \%$ de la biomasa, con las siguientes contribuciones: Aphanocapsa incerta (40.2\%), Coelosphaerium aerugineum (24\%), Snowella lacustris (15.3\%), Merismopedia spp. (10.7\%) y Aphanocapsa spp. (6.62\%). El 3 de abril de 2015, los dos taxones con mayor contribución fueron Aphanocapsa incerta (64.8\%) y Aphanocapsa spp. (31.4\%). En cuanto al pico de la densidad, correspondió a Merismopedia spp. con el 96.3\%. (Figura 3b).

En Centenario, Bacillarophyceae presentó su mayor biovolumen entre el 24 de enero de 2014 y el 3 de abril de 2015. En ese período, Fragilaria acus representó el $96.7 \%$ del total del biovolumen. La mayor biomasa de Cyanobacteria (registrada entre el 7 de febrero de 2014 y el 18 de abril de 2015) correspondió a Aphanocapsa spp. con el 97.2\%. (Figura 3a).

En Regatas, Fragilaria acus (Bacillarophyceae) constituyó un 88.3\% (biovolumen) del 3 de septiembre al 3 de octubre de 2014. La clase Cryptophyceae estuvo presente durante todo el período de muestreo, y Cryptomonas marssonii (50.6\%) y C. ovata (36.8\%) fueron sus principales representantes. En lo que respecta a Chlorophyceae, las más relevantes entre el 18 de noviembre de 2014 y el 10 de enero de 2015 fueron Coelastrum astroideum (64.7\%) y Pediastrum boryanum (6.7\%) (Figura 3a).

Grupos funcionales. En el caso del lago del Rosedal, 5 codones del sistema de clasificación de Reynolds, actualizado por Padisák, constituyeron el 94.6\% del total del biovolumen (K, L0, C, F, J). También 5 codones de la clasificación de Reynolds fueron dominantes en los otros dos lagos. En Centenario conformaron el $92.3 \%$ del biovolumen (D, K, J, P, X2) y en Regatas, el 92.1\% (D, Y, J, P, X2) (Figura 4a, b, c).
Tomando en cuenta la clasificación de Kruk, en Rosedal, 4 codones (VII, IV, VI, V) representaron el $97.9 \%$ del biovolumen. Del mismo modo, 4 codones fueron los más importantes en los otros dos lagos: en Centenario constituyeron el $98.2 \%$ del biovolumen (VI, VII, IV, V), y el $98.4 \%$ del biovolumen (VI, V, IV, VII) en Regatas (Figura $4 a, b, c)$.

Composición de especies. De acuerdo con el análisis cualitativo microscópico de las muestras de fitoplancton se identificaron en total 170 especies en Regatas, 134 en Rosedal y 93 en Centenario (Tabla 4). A partir del diagrama de Olmstead-Tukey se puede analizar de forma conjunta la densidad de las especies o géneros, y su frecuencia de aparición en todo el período de muestreo. De esta manera, en Centenario fueron dominantes, muy abundantes y frecuentes (81-100\%) Fragilaria acus, Monoraphidium komarkovae, Pseudostaurosira brevistriata, Chaetoceros sp. y Nitzschia gracilis. Abundantes y frecuentes (63-75\%) Chroomonas sp., Scenedesmus opoliensis, Monoraphidium contortum y Fragilaria construens. Ocasionales y abundantes Aphanocapsa spp. y Ochromonas sp. (Figura 5a). En Regatas dominaron Plagioselmis lacustris, Cryptomonas marssonii y Crytomonas ovata (muy abundantes, frecuencia relativa: 94$100 \%$ ). Abundante: Fragilaria acus, con un $63 \%$ de frecuencia relativa. Ocasional y abundante: Merismopedia tenuissima (Figura 5b). En Rosedal dominaron Merismopedia spp., Aphanocapsa spp., Cyclotella spp., Monoraphidium contortum (muy abundantes, con una frecuencia relativa del 100\%), Nitzschia palea, N. acicularis, Aphanocapsa incerta, Aphanothece spp., Tetraedrom minimum, Anabaenopsis circularis, Pseudostaurosira brevistriata (abundantes, con una frecuencia relativa entre el 81-100\%). Ocasionales y abundantes: Coelosphaerium aerugineum, Dictyosphaerium sp., Chroomonas sp. y Actinastrum hantzschii (Figura 5c).

\section{Análisis multivariados}

Ambos análisis (CCA y RDA) basados en biovolumen de los grupos funcionales arrojaron un ordenamiento muy similar al obtenido con el biovolumen de especies. Los resultados muestran una clara separación de los tres lagos urbanos. Las variables con mayor peso en estos análisis fueron las mismas (Figura 6a, b, c).

CCA basado en biovolumen de especies. La varianza acumulada de la relación biovolumen 

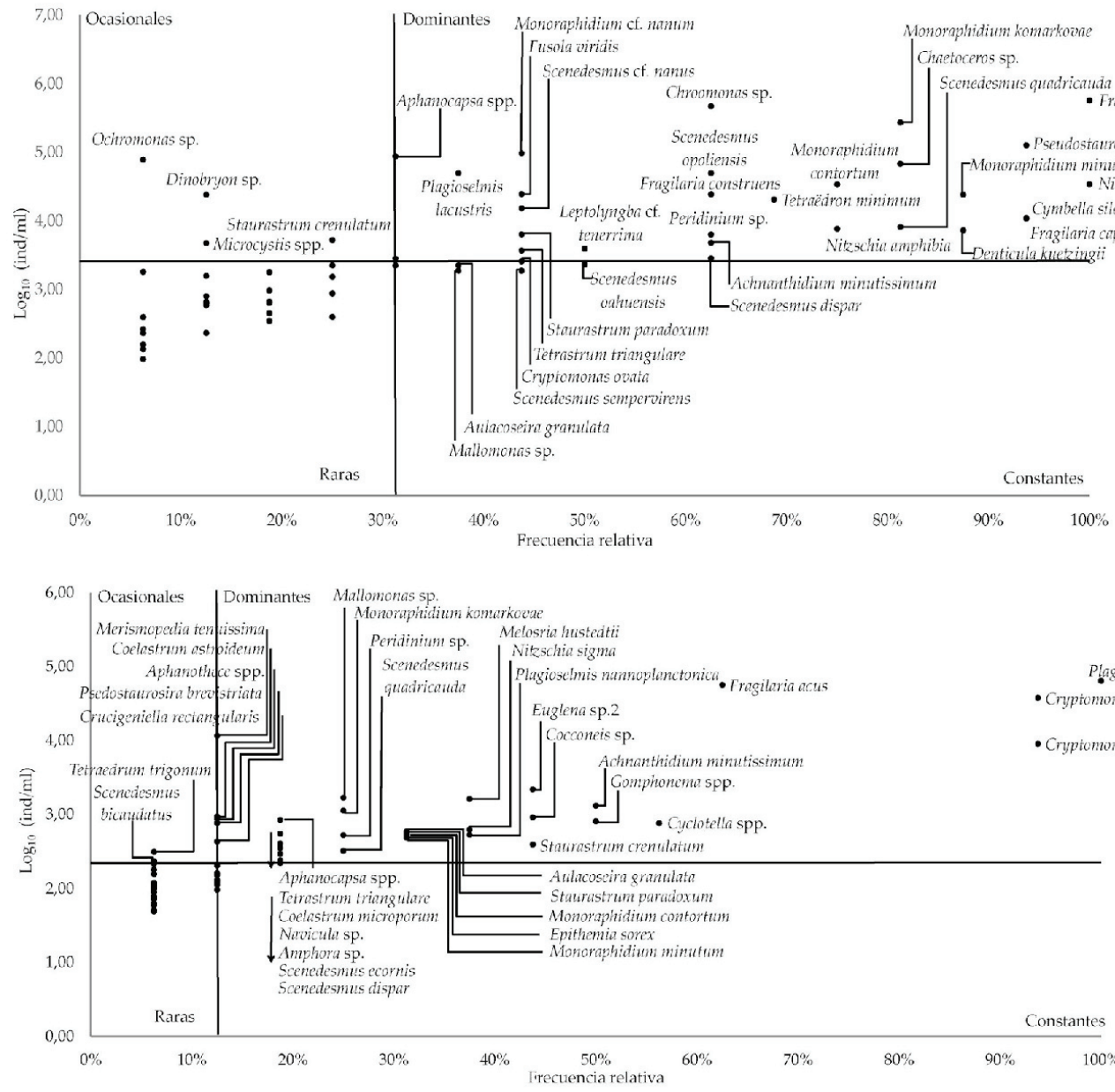

a)

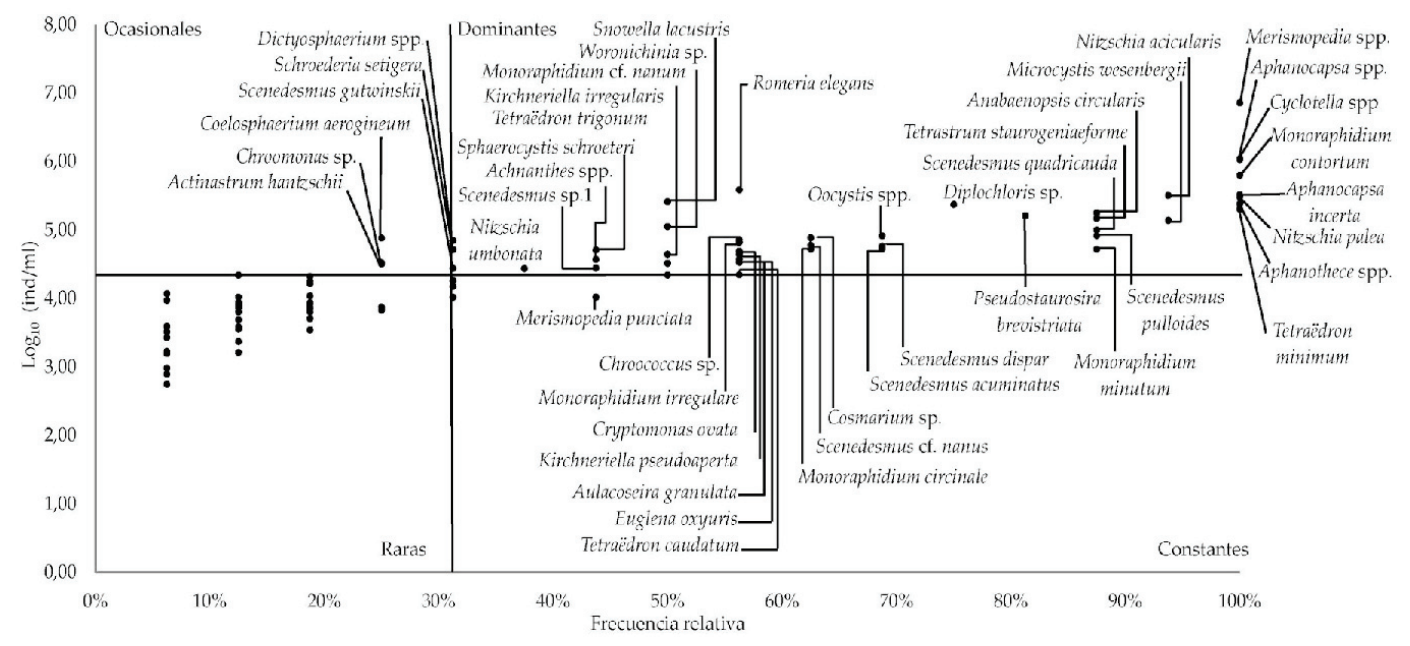

Figura 5. Diagrama de Olmstead-Tukey de la densidad (individuos $/ \mathrm{mL}$ ) de cada especie o género registrada en cada lago urbano: a) Centenario, b) Regatas, c) Rosedal, de septiembre de 2014 a abril de 2015.

Figure 5. Olmstead-Tukey diagram of the density (individuals $/ \mathrm{mL}$ ) of each species and/or gender registered in each urban lake: a) Centenario, b) Regatas, c) Rosedal, from September 2014 to April 2015. 
Tabla 4. Lista taxonómica de algas registradas en cada lago urbano (CE: Centenario, RE: Regatas, RO: Rosedal) de septiembre de 2014 a abril de 2015. X: presente. Reynolds: grupos funcionales de la clasificación de Reynolds et al. (2002), actualizada por Padisák et al. (2009). Kruk: grupos funcionales basados en aspectos morfológicos de Kruk et al. (2010).

Table 4. Taxonomic list of algae registered in each urban lake (CE: Centenario, RE: Regatas, RO: Rosedal) from September 2014 to April 2015. X: present. Reynolds: functional groups of the Reynolds et al. (2002) classification, updated by Padisák et al. (2009). Kruk: functional groups based on morphological aspects of Kruk et al. (2010).

\begin{tabular}{llllll}
\hline Taxa & Reynolds & Kruk & CE & RE & RO \\
Riqueza total & & & 93 & 170 & 134
\end{tabular}

Cyanobacteria

Anabaenopsis circularis

Aphanocapsa delicatissima

Aphanocapsa elachista

Aphanocapsa holsatica

Aphanocapsa incerta

Aphanocapsa spp.

Aphanothece nidulans

Aphanothece spp.

Chroococcus dispersus

Chroococcus minimus

Chroococcus minutus

Chroococcus sp.

Coelosphaerium aerugineum

Dolichospermum sp.

Leptolyngbya cf. tenerrima

Merismopedia minima

93

Merismopedia punctata

Merismopedia tenuissima

Merismopedia sp.

Microcystis cf. flosaquae

Microcystis wesenbergii

Microcystis spp.

Phormidium aerugineo-coeruleum

Phormidium sp.

Pseudanabaena catenata

Rhabdoderma lineare

Romeria elegans

Snowella lacustris

Woronichinia sp.

$\begin{array}{cc}\text { H1 } & \text { III } \\ \text { K } & \text { VII } \\ \text { K } & \text { VII } \\ \text { K } & \text { VII } \\ \text { K } & \text { VII } \\ \text { K } & \text { VII } \\ \text { K } & \text { VII } \\ \text { K } & \text { VII } \\ \text { L0 } & \text { VII } \\ \text { L0 } & \text { I } \\ \text { L0 } & \text { VII } \\ \text { L0 } & \text { VII } \\ \text { K } & \text { IV } \\ \text { H1 } & \text { III } \\ \text { S1 } & \text { IV } \\ \text { L0 } & \text { I } \\ \text { L0 } & \text { VII } \\ \text { L0 } & \text { VII } \\ \text { L0 } & \text { VII } \\ \text { M } & \text { VII } \\ \text { M } & \text { VII } \\ \text { M } & \text { VII } \\ \text { S1 } & \text { IV } \\ \text { S1 } & \text { IV } \\ \text { S1 } & \text { III } \\ \text { MP } & \text { IV } \\ \text { MP } & \text { III } \\ \text { L0 } & \text { VII } \\ \text { L0 } & \text { VII } \\ & \end{array}$

$x$

VII

VII

VII

$\mathrm{X}$

$X$

Actinastrum hantzschii var. hantzschii
Actinastum hanchil

Actinastrum hantzschii var. subtile

Ankistrodesmus densus

Ankistrodesmus fusiformis

Ankistrodesmus gracilis

Ankistrodesmus sigmoides

Coelastrum astroideum

Coelastrum microporum

Coelastrum pseudomicroporum

Coenochloris mucosa

Crucigeniella crucífera

Crucigeniella neglecta

Crucigeniella rectangularis

Crucigeniella sp.

Dictyosphaerium chlorelloides

Dictyosphaerium pulchellum

Dictyosphaerium spp.

Diplochloris decusata

Diplochloris sp.

Fusola viridis

Golenkiniopsis solitaria

Keratococcus bicaudatus

Keratococcus raphidioides

Kirchneriella aperta

Kirchneriella contorta

Kirchneriella irregularis var. irregularis

Kirchneriella irregularis var. spiralis

Kirchneriella obesa

Kirchneriella pinguis

Kirchneriella pseudoaperta

Lobocystis planctónica

Lobocystis sp.

X

$\begin{array}{cc}X & X \\ X & X \\ & X \\ X & X \\ & X \\ X & X \\ & X \\ X & \\ X & \end{array}$

$\underset{X}{X}$

$X$

$\mathrm{X}$

$X$
$X$

$\mathrm{X}$

$X$

VII 
Tabla 4. Continuación.

Table 4. Continuation

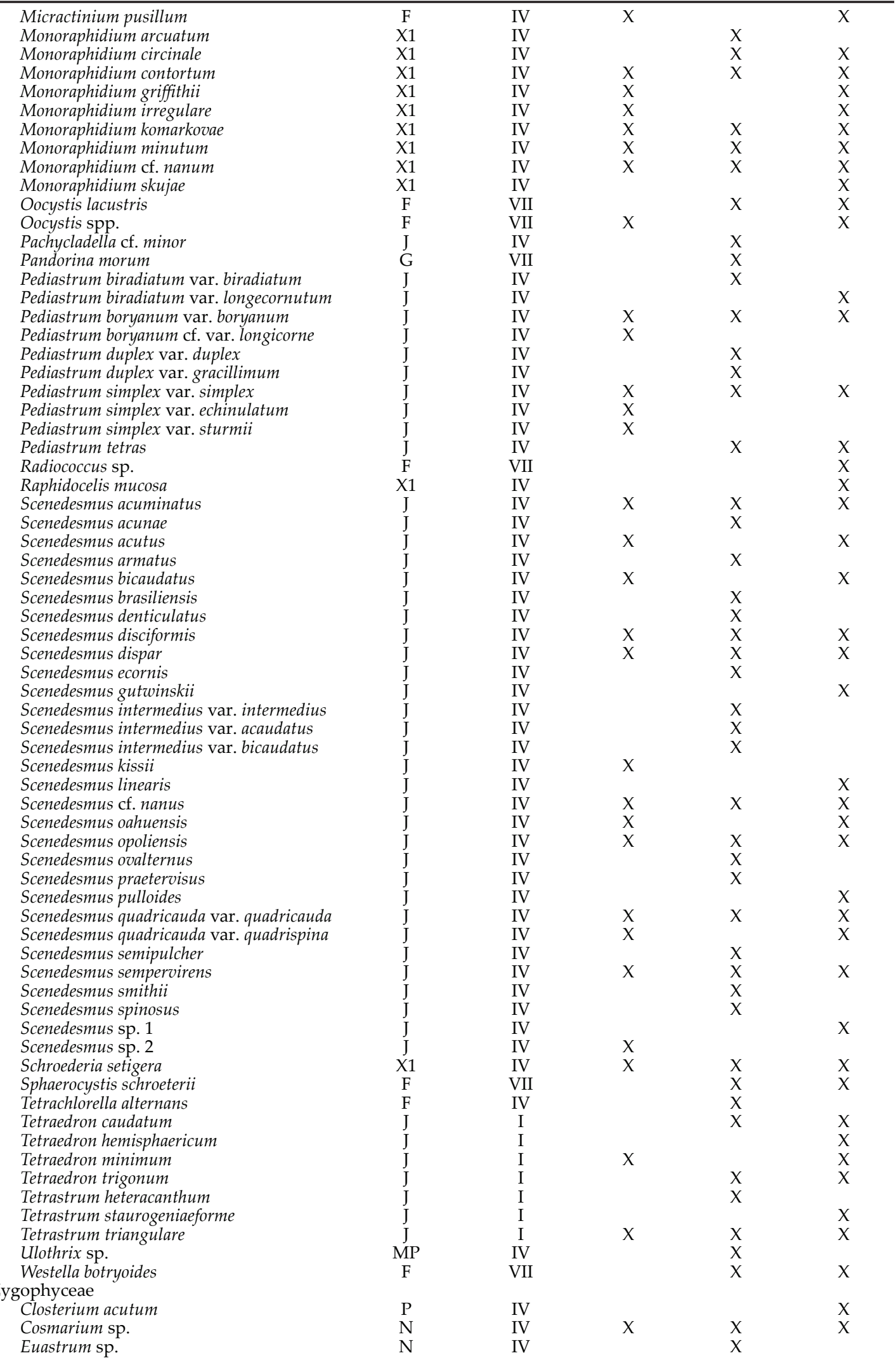


DiNÁMICA DEL FITOPLANCTON EN LAGOS URBANOS

Tabla 4. Continuación.

Table 4. Continuation

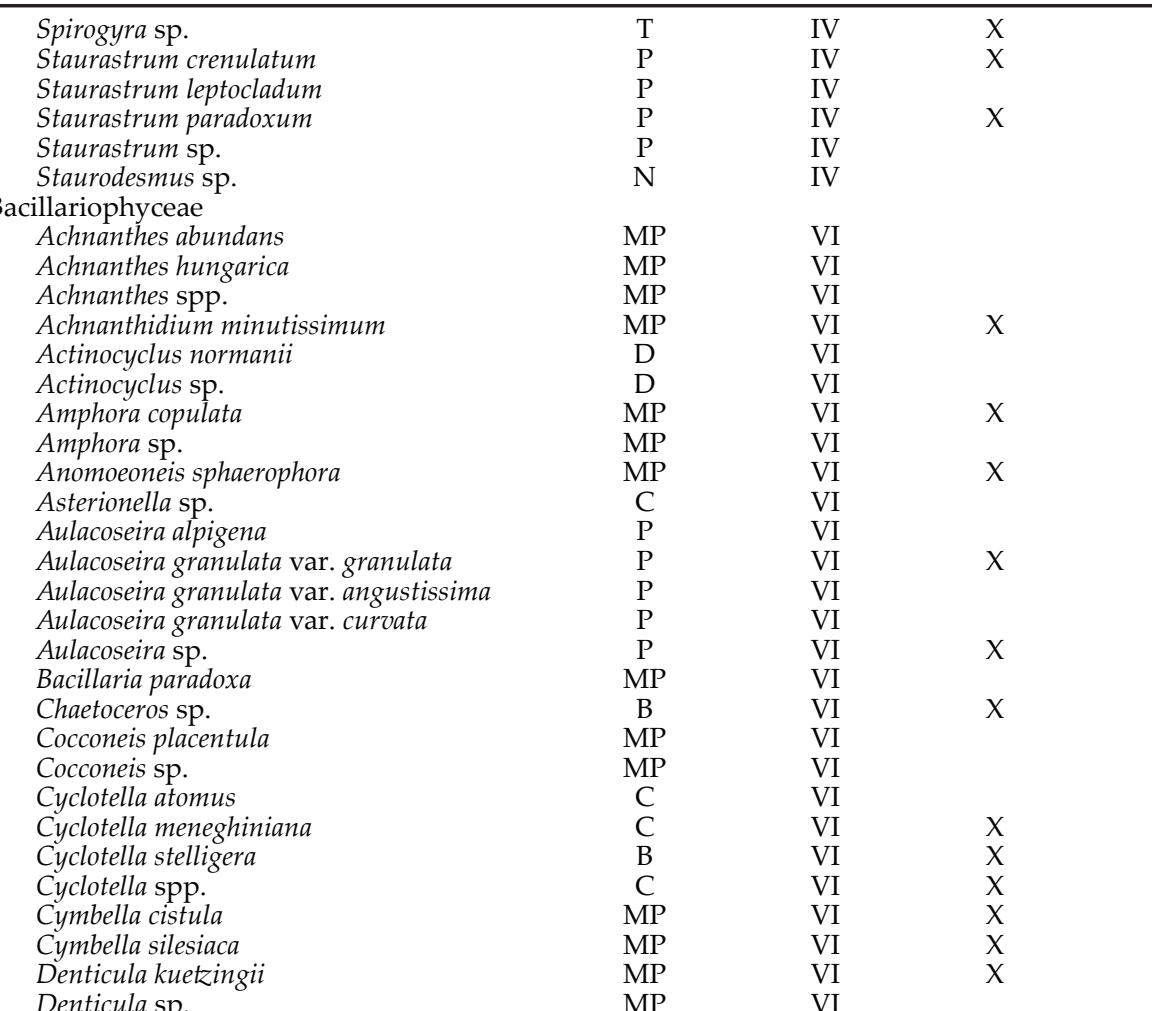

$\mathrm{X}$

X

$x$

$\mathrm{X}$

$x$

$x$

$x \quad x$

$X$

$X$

$\mathrm{X}$

$x$

$\mathrm{X}$

X

X

$X$
$X$
$X$

$x$

$x$

$X$

$X$
$X$

$x$

$\mathrm{X}$

X

$X$
$X$
$X$
$X$
$X$
$X$

$X$

$\mathrm{X}$

Diploneis finnica

Diploneis sp.

Epithemia adnata

Epithemia sorex

Eunotia implicata

Eunotia monodon

Eunotia pectinalis

Eunotia sp.

Fragilaria acus

Fragilaria capucina

Fragilaria construens

Fragilaria pulchella

Fragilaria sp.

Gomphonema affine

Gomphonema clavatum

Gomphonema clevei

Gomphonema constrictum

Gomphonema parvulum

Gomphonema truncatum

Gomphonema spp.

Gyrosigma attenuatum

Gyrosigma sp.

Hantzschia elongata

Hantzschia sp.

Melosira hustedtii

Melosira varians

Melosira sp.

Navicula cryptocephala

Navicula cuspidata

Navicula hungarica

Navicula perrotettii

Navicula veneta

Navicula sp.

Neidium ampliatum

Neidium sp.

Nitzschia acicularis

Nitzschia amphibia

Nitzschia angustata
D

$\mathrm{MP}$

MP

MP VI

MP VI

MP VI

D VI

MP VI

MP VI

MP VI

MP VI

MP VI

MP $\quad$ VI

MP VI

MP VI

MP $\quad$ VI

MP VI

MP $\quad$ VI

$\begin{array}{cc}M P & \text { VI } \\ D & \text { VI }\end{array}$

VI

P VI

P VI

VI

MP VI

MP VI

MP $\quad$ VI

$\begin{array}{ll}\text { MP } & \text { VI } \\ \text { MP } & \text { VI }\end{array}$

MP $\quad$ VI

MP VI

MP VI

D $\quad$ VI

$\begin{array}{ll}\mathrm{D} & \text { VI } \\ \mathrm{D} & \text { VI }\end{array}$ 
Tabla 4. Continuación.

Table 4. Continuation

\begin{tabular}{|c|c|c|c|c|c|}
\hline Nitzschia constricta & $\mathrm{D}$ & VI & & $x$ & \\
\hline Nitzschia dissipata & $\mathrm{D}$ & VI & $\mathrm{x}$ & & \\
\hline Nitzschia frustulum & $\mathrm{D}$ & VI & & $x$ & \\
\hline Nitzschia gracilis & $\mathrm{D}$ & VI & $x$ & & $X$ \\
\hline Nitzschia hungarica & $\mathrm{D}$ & VI & & $x$ & \\
\hline Nitzschia levidensis & $\mathrm{D}$ & VI & & $x$ & \\
\hline Nitzschia linearis & $\mathrm{D}$ & VI & & $x$ & \\
\hline Nitzschia palea & $\mathrm{D}$ & VI & & $x$ & $x$ \\
\hline Nitzschia reversa & $\mathrm{D}$ & VI & & & $X$ \\
\hline Nitzschia scalaris & $\mathrm{D}$ & VI & & $x$ & \\
\hline Nitzschia sigma & $\mathrm{D}$ & VI & $x$ & $x$ & $\mathrm{X}$ \\
\hline Nitzschia sigmoidea & $\mathrm{MP}$ & VI & $\mathrm{X}$ & & \\
\hline Nitzschia tryblionella & $\mathrm{D}$ & VI & & $x$ & \\
\hline Nitzschia umbonata & $\mathrm{D}$ & VI & $x$ & $x$ & $\mathrm{X}$ \\
\hline Nitzschia vermicularis & $\mathrm{D}$ & VI & & $x$ & \\
\hline Nitzschia spp. & $\mathrm{D}$ & VI & & $x$ & $\mathrm{X}$ \\
\hline Pinnularia maior & $\mathrm{MP}$ & VI & & $x$ & \\
\hline Pinnularia mesolepta & $\mathrm{MP}$ & VI & & $x$ & \\
\hline Pinnularia microstauron & MP & VI & & $x$ & \\
\hline Pinnularia viridis & MP & VI & $\mathrm{x}$ & & \\
\hline Pinnularia sp. & MP & VI & & $x$ & \\
\hline Plagiotropis lepidoptera & MP & VI & & $X$ & \\
\hline Pseudostaurosira brevistriata & $\mathrm{P}$ & VI & $\mathrm{x}$ & $x$ & $x$ \\
\hline Stauroneis sp. & $\mathrm{MP}$ & VI & & $x$ & \\
\hline Surirella angustata & MP & VI & & $x$ & \\
\hline Surirella robusta & MP & VI & $x$ & $x$ & \\
\hline Surirella tenera & MP & VI & $\mathrm{X}$ & $x$ & $\mathrm{X}$ \\
\hline Surirella sp. & $\mathrm{MP}$ & VI & & $x$ & \\
\hline \multicolumn{6}{|l|}{ Chrysophyceae } \\
\hline Chroomonas sp. & $\mathrm{X} 2$ & $\mathrm{~V}$ & $\mathrm{x}$ & & $\mathrm{X}$ \\
\hline Dinobryon sp. & $\mathrm{E}$ & II & $x$ & $x$ & $x$ \\
\hline Mallomonas sp. & $\mathrm{E}$ & II & $\mathrm{X}$ & $\mathrm{X}$ & $x$ \\
\hline Ochromonas sp. & $\mathrm{X} 2$ & II & $\mathrm{X}$ & & $\mathrm{X}$ \\
\hline \multicolumn{6}{|l|}{ Cryptophyceae } \\
\hline Cryptomonas marssonii & Y & $\mathrm{V}$ & $\mathrm{x}$ & $x$ & $\mathrm{X}$ \\
\hline Cryptomonas ovata & $\mathrm{Y}$ & $\mathrm{V}$ & $\mathrm{x}$ & $x$ & $\mathrm{X}$ \\
\hline Plagioselmis lacustris & $\mathrm{X} 2$ & $\mathrm{~V}$ & $\mathrm{X}$ & $x$ & $\mathrm{X}$ \\
\hline Plagioselmis nannoplanctonica & $\mathrm{X} 2$ & $\mathrm{~V}$ & & $x$ & \\
\hline \multicolumn{6}{|l|}{ Euglenophyceae } \\
\hline Euglena allorgei & W1 & $\mathrm{V}$ & & $x$ & \\
\hline Euglena oxyuris & W1 & $\mathrm{V}$ & & $x$ & $\mathrm{X}$ \\
\hline Euglena sp. 1 & W1 & $\mathrm{V}$ & & & $\mathrm{X}$ \\
\hline Euglena sp. 2 & W1 & $\mathrm{V}$ & & $x$ & \\
\hline Euglena sp. 3 & W1 & $\mathrm{V}$ & & $x$ & $x$ \\
\hline Lepocinclis ovum var. ovum & W1 & $\mathrm{V}$ & & $x$ & \\
\hline Lepocinclis ovum var. ecaudata & W1 & $\mathrm{V}$ & & $x$ & \\
\hline Lepocinclis salina & W1 & $\mathrm{V}$ & & $x$ & \\
\hline Lepocinclis texta & W1 & $\mathrm{V}$ & & $X$ & \\
\hline Phacus orbicularis & W1 & $\mathrm{V}$ & & $x$ & \\
\hline Phacus pseudonordstedtii & W1 & $\mathrm{V}$ & & $x$ & \\
\hline Trachelomonas sp. & W2 & $\mathrm{V}$ & & $x$ & \\
\hline Strombomonas scabra & W2 & $\mathrm{V}$ & & $x$ & \\
\hline Strombomonas verrucosa & W2 & $\mathrm{V}$ & $\mathrm{x}$ & $x$ & \\
\hline Strombomonas sp. & W2 & $\mathrm{V}$ & & & $\mathrm{X}$ \\
\hline \multicolumn{6}{|l|}{ Dinophyceae } \\
\hline Ceratium furcoides & L0 & $\mathrm{V}$ & & $x$ & \\
\hline Peridinium cf. umbonatum & L0 & $\mathrm{V}$ & $\mathrm{x}$ & & \\
\hline Peridinium sp. & L0 & $\mathrm{V}$ & $x$ & $x$ & $\mathrm{X}$ \\
\hline \multicolumn{6}{|l|}{ Xanthophyceae } \\
\hline Goniochloris fallax & $\mathrm{J}$ & I & & $x$ & \\
\hline Goniochloris sp. & $\mathrm{J}$ & $\mathrm{I}$ & & $x$ & \\
\hline
\end{tabular}

de especies-variables ambientales del primer y segundo eje representó el 77.7\% (eje 1: 47.8\%). De acuerdo con la prueba de Monte Carlo, las variables ambientales se correlacionaron significativamente con el primer eje $(P$ - valor $=0.002)$; también fue significativa la prueba de todos los ejes ( $P$-valor $=0.002)$. En relación con la matriz de correlación ponderada, el primer eje mostró una mayor correlación con el nitrógeno total (0.75), la 

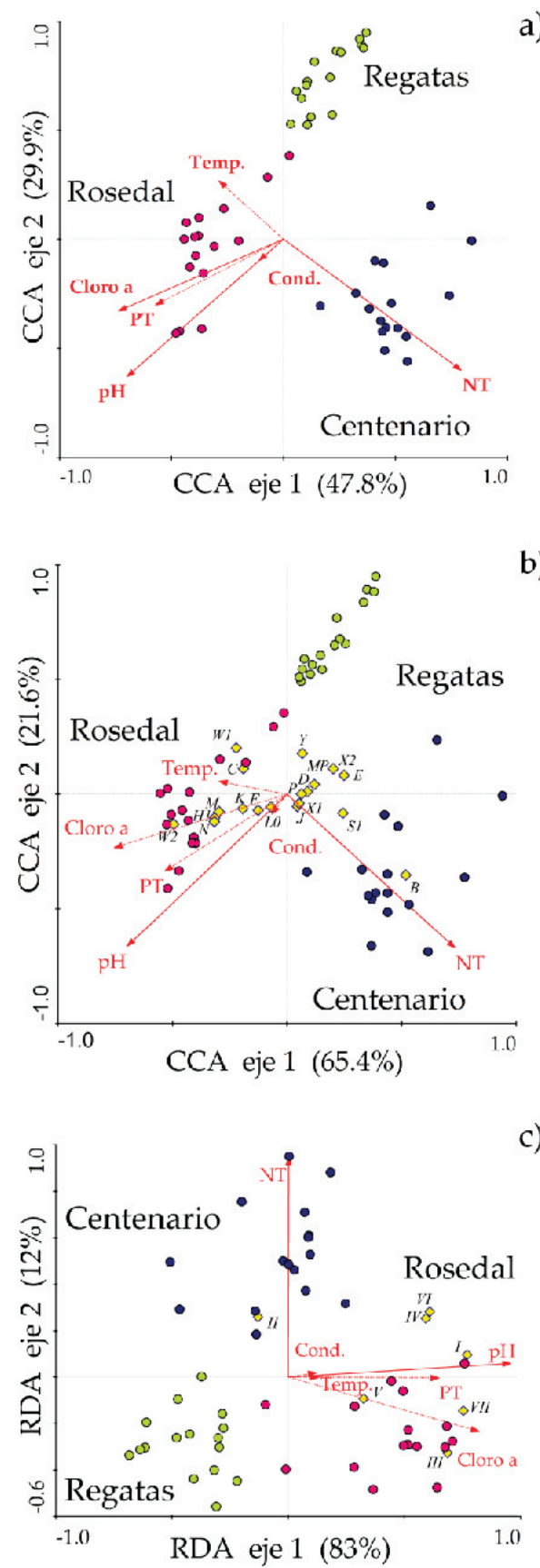

Figura 6. a) Biplot del CCA basado en el biovolumen de las especies; b) triplot del CAA basado en biovolumen de grupos funcionales de Reynolds et al. (2002); c) RDA basado en el biovolumen de grupos morfo-funcionales de Kruk et al. (2010). Las variables ambientales significativas $(P<0.05)$ se indican con vectores de trazo continuo. Temp.: temperatura del agua; Cloro a: clorofila a; NT: nitrógeno total; PT: fósforo total; Cond.: conductividad. Figure 6. a) Biplot of the CCA based on species biovolume; b) triplots of the CCA based on the biovolume of functional groups proposed by Reynolds et al. (2002); c) triplot of the RDA based on biovolume of morpho-functional groups of Kruk et al. (2010). Significant environmental variables $(P<0.05)$ are indicated with continuous line vectors. Temp.: water temperature; Cloro a: chlorophyll a; NT: total nitrogen; PT: total phosphorus; Cond.: conductivity. clorofila $a(-0.70)$, el pH (-0.66) y el fósforo total $(-0.54)$; el segundo eje se correlacionó con el $\mathrm{pH}(-0.54)$ y el nitrógeno total (-0.52).

En el biplot de muestras y variables ambientales se observa que los tres lagos se separan claramente en el espacio. Las muestras de Centenario se ordenan hacia valores crecientes de nitrógeno total, las del Rosedal hacia mayores valores de clorofila $a, \mathrm{PT}, \mathrm{pH}$, temperatura y conductividad, mientras que las muestras de Regatas se ubican en la parte superior del gráfico, siguiendo un patrón opuesto al del Rosedal (menores valores de clorofila $a, \mathrm{PT}$ y pH). (Figura 6a).

CCA basado en biovolumen de grupos funcionales de Reynolds. En el CCA basado en la clasificación de Reynolds (Figura 6b), los dos primeros ejes explicaron el $87 \%$ de la varianza (eje 1: 65.4\%). La prueba de Monte Carlo indicó en ambos casos que las variables ambientales se correlacionaron significativamente con el primer eje $(P$-valor=0.002), siendo también significativa la prueba de todos los ejes $(P$ valor=0.002).

Acerca de la matriz de correlación ponderada, para los grupos de Reynolds el primer eje se correlacionó con la clorofila $a(-0.68)$, el pH ($0.63)$, el fósforo total (-0.48) y el nitrógeno total (0.66); el segundo eje se correlacionó con el pH $(-0.49)$ y con el nitrógeno total (-0.49).

$\underline{\text { RDA basado en biovolumen de grupos }}$ funcionales de Kruk. En el caso del biovolumen de grupos funcionales de Kruk (Figura 6c), el mejor análisis a aplicar por la distribución de los datos fue el análisis de redundancia (RDA), cuyos dos primeros ejes explicaron un 95\% de la variabilidad total (eje 1: 83\%). En relación con la matriz de correlación ponderada, el primer eje mostró una mayor correlación con el pH (0.84), la clorofila a (0.72) y el fósforo total (0.57); el segundo eje se correlacionó con el nitrógeno total (0.66).

\section{DisCUSIÓN}

Los resultados de este trabajo evidenciaron diferencias marcadas entre los tres lagos urbanos estudiados, tanto en sus características limnológicas como en el estado trófico. Teniendo en cuenta las variables más importantes vinculadas al trofismo (i.e., clorofila $a$ fitoplanctónica y fósforo total), se puede clasificar al lago del Rosedal como hipereutrófico, a Centenario como eutrófico, y a Regatas como meso-eutrófico. Los tres 
ambientes también difirieron de forma marcada en la estructura de sus comunidades fitoplanctónicas, tanto en la composición de especies como en sus grupos funcionales.

Como ya se señaló en numerosas investigaciones sobre lagos urbanos (e.g., Britton et al. 1977; Waajen et al. 2016), estos ambientes tienden a eutrofizarse rápidamente. Si se tiene en cuenta que la eutrofización es el principal problema que afecta la calidad del agua de los sistemas lénticos a nivel global, las estrategias de mitigación se deben definir para cada ambiente acuático en particular a partir del análisis de la composición biológica y de la estimación de los flujos de nutrientes (Lürling et al. 2016).

Las escasas acciones de manejo que se llevan a cabo en el lago Rosedal, los tiempos largos de retención del agua, junto con el enriquecimiento de las aguas por fósforo son condiciones que favorecen la proliferación de las cianobacterias, en particular durante el período cálido. Estas condiciones se suman a la falta de macrófitas sumergidas, lo que favorece la resuspensión de sedimentos y la estabilización del cuerpo de agua en un estado de aguas turbias (Søndergaad et al. 2001 y citas en este trabajo). No obstante, también sería necesario obtener información sobre la abundancia y la composición del zooplancton a fin de evaluar si esta comunidad podría ejercer un control adecuado de tipo top-down sobre el fitoplancton.

De acuerdo con la clasificación de Kruk et al. (2010), en el lago Rosedal, el grupo funcional fitoplanctónico más importante fue el VII, que incluye Chroococcales y Oscillatoriales; algunas especies de este grupo tienen propiedades boyantes y pueden convertirse en un problema serio si producen toxinas y forman espumas sobre la superficie. Si consideramos la clasificación de Reynolds et al. (2002), los grupos predominantes fueron el $\mathrm{K}$ (asociado con hábitats poco profundos y con columnas de aguas ricas en nutrientes) y el L0, que es usual en lagos poco profundos (oligo a eutróficos). Los dos grupos son susceptibles a la mezcla profunda o prolongada.

En el lago Rosedal, las floraciones de cianobacterias son recurrentes y ya habían sido reportadas para el cuerpo de agua desde hace décadas (Izaguirre et al. 1986). Las floraciones de cianobacterias pueden generar diversos efectos negativos sobre la red trófica; entre ellos, al aumentar la turbidez imposibilitan el desarrollo de plantas acuáticas sumergidas, afectando el hábitat de muchos invertebrados y peces. Por otro lado, las floraciones pueden producir una depleción de oxígeno durante la noche, lo cual puede causar la muerte de peces (Paerl and Huisman 2009). Algunas producen varios tipos de toxinas que pueden constituir un riesgo para las aves, los seres humanos y las mascotas (Backer 2002; Paerl et al. 2011; Kruk et al. 2015). De acuerdo con la Organización Mundial de la Salud (WHO 2003), existe una probabilidad alta de efectos adversos para la salud cuando ocurre una exposición a una concentración de más de 100000 células/mL de cianobacterias o mayor a $50 \mu \mathrm{g} / \mathrm{L}$ de clorofila $a$ con dominancia de cianobacterias. Los resultados obtenidos en nuestro estudio muestran que éste sería el caso del lago Rosedal, y por ello se sugiere iniciar acciones de manejo que reduzcan el riesgo por exposición. Algunas de posibles acciones serían: la reducción de la carga externa de nutrientes (prevención del ingreso por escorrentía), con un especial interés en el fósforo, y la mezcla artificial del lago (profunda y prolongada) para romper la estabilidad de la columna de agua de la cual las cianobacterias dependen (Mantzouki et al. 2016, y citas en sus fuentes). Por otro lado, en sistemas hipereutróficos como el lago Rosedal, el restablecimiento de vegetación sumergida es una medida a largo plazo que también se podría implementar en el centro del lago, donde el cuerpo de agua tiene menor profundidad. El restablecimiento de la comunidad de plantas acuáticas es una medida de restauración que suele mejorar la calidad del agua y disminuir el riesgo de floraciones (e.g., Qiu, et al. 2001; Moss 2007; Scheffer and Jeppesen 2007). Además, las plantas acuáticas proveen una estructura del hábitat que influye en la comunidad de peces, lo que permite que el zooplancton y los macroinvertebrados ejerzan un control de arriba hacia abajo sobre las algas, control que, en gran medida, es independiente de la carga de nutrientes (Phillips et al. 2016, y citas en sus fuentes). No obstante, si bien el restablecimiento de las macrófitas puede ser una medida beneficiosa, las macrófitas también pueden generar posibles conflictos para los usuarios recreativos cuando las plantas se vuelven muy abundantes y molestan la navegación u obstruyen el flujo de agua (Jeppesen et al. 2012). Por esta razón, las plantas en este lago hipereutrófico deberían ser cosechadas de manera periódica.

Además, las acciones de manejo deberían incluir no sólo la reducción de la carga externa 
de fósforo, sino también la mitigación de los efectos del ciclo interno de este nutriente desde los sedimentos. En este sentido, en lagos eutróficos relativamente pequeños con floraciones de cianobacterias, el cambio de un estado de aguas turbias a aguas claras se puede generar trabajando sobre la columna de agua y los sedimentos mediante el uso de floculantes de P y sorbentes de fase sólida (e.g., Hansen and Márquez-Pacheco 2012; Lürling and van Oosterhout 2013; Lürling et al. 2016). En muchos casos es recomendable utilizar métodos combinados de restauración (fisicoquímicos y biológicos) para mejorar la estabilidad a largo plazo y, de esta manera, reducir los costos de restauración (Jeppesen et al. 2012 y citas en este trabajo). Para Sudamérica se puede mencionar como ejemplo de restauración de un lago urbano la realizada en el lago Rodó (Uruguay) (Scasso et al. 2001), que incluyó primeramente un drenaje con remoción de sedimentos, desviación de afluentes y llenado con agua subterránea, y posteriormente el diseño de un sistema de bombeo con circulado del agua a través de piscinas con vegetación flotante. Sin embargo, para garantizar la efectividad a largo plazo de las medidas de manejo es importante que éstas se mantengan en el tiempo, sobre todo teniendo en cuenta que en un contexto urbano las perturbaciones son permanentes.

Por otro lado, los resultados de nuestras investigaciones evidenciaron que el lago de Regatas se encuentra en un estado de aguas claras. Los niveles de fósforo total fueron los más bajos con respecto a los otros dos estanques, debidoprobablementea la presencia de plantas sumergidas durante el período del presente estudio. Al producir oxigenación en el fondo, las plantas aumentan las reacciones redox, lo que facilita la absorción de fósforo por los compuestos de hierro y disminuye la resuspensión de sedimentos (Søndergaad et al. 2001). Además, la vegetación genera una supresión de crecimiento algal debido a la reducción de nutrientes disponibles, la producción de sustancias alelopáticas tóxicas para las algas (Scheffer et al. 1993) $y$, eventualmente, la provisión de refugios para el zooplancton herbívoro. Sumado a la presencia beneficiosa de las macrófitas, el lago de Regatas tiene un sistema de recirculación de agua que da la opción de manejar los tiempos de retención ante un escenario de posibles floraciones de cianobacterias.

En lo que respecta a los grupos funcionales del fitoplancton, en Regatas, según la clasificación de Kruk, dominó el grupo VI (organismos sin flagelos, con exoesqueleto de sílice), seguido por el V (Cryptophyceae, entre otros). Teniendo en cuenta la clasificación de Reynolds, dominó el codón D, que incluye diatomeas de los géneros Synedra/Fragilaria, Nitzschia y algunas diatomeas céntricas; este grupo se asocia con aguas turbias poco profundas, es vulnerable al agotamiento de nutrientes y tolerante al lavado. El codón Y fue codominante e incluye Cryptomonas grandes que se encuentran en un amplio rango de hábitats, generalmente en lagos pequeños enriquecidos. La presencia conjunta de los codones D e $\mathrm{Y}$ coincide con lo reportado por Avigliano et al. (2014) para la laguna Los Coipos, de la Reserva Costanera Sur de CABA. En otras lagunas de esta misma reserva, Sinistro et al. (2013) observaron que el crecimiento de Cryptomonas marssonii (estratega r) estuvo relacionado con bajos niveles de conductividad, lo que concuerda con lo hallado en Regatas, en donde esta especie se asoció a niveles bajos de conductividad, siendo dominante y frecuente.

Con respecto al lago Centenario, sus características limnológicas, la dominancia de diatomeas y la alta frecuencia de crisófitas registradas concuerdan con las descripciones reportadas en un informe técnico previo de este lago realizado en 2012 (Izaguirre and Sinistro, datos no publicados). En este informe se informaron también valores elevados de nitratos y de silicatos. Estos últimos duplicaron o triplicaron las concentraciones medias de dióxido de silicio consideradas como usuales en aguas continentales superficiales. El sílice es importante para el desarrollo de diatomeas y crisófitas (en estas últimas para la producción de quistes). En el estudio de 2012 se reportó una floración de Nitzschia gracilis, acompañada por una elevada densidad de Fragilaria acus y se clasificó al cuerpo de agua como hipereutrófico. En el presente trabajo, las variables de trofismo indicarían un estado eutrófico; el cambio podría atribuirse a las acciones de manejo implementadas, tales como el recirculado del agua y la disposición de filtros. Cabe señalar que los altos niveles de nitrógeno total y de conductividad registrados en este lago estarían vinculados al ingreso de agua subterránea. Esta suposición se basa en registros obtenidos en 2011 en dos estaciones subterráneas cercanas al lago Centenario (GCABA-F046 y la GCABAF051), correspondientes al acuífero superficial Pampeano-Postpampeano; en las mismas se 
hallaron concentraciones de nitratros de $15 \mathrm{mg} / \mathrm{L}$ y $131 \mathrm{mg} / \mathrm{L}$ respectivamente, y conductividades de $915 \mu \mathrm{S} / \mathrm{cm}$ y de 1380 $\mu \mathrm{S} / \mathrm{cm}$ (datos Acumar).

Con respecto a los grupos funcionales fitoplanctónicos, según la clasificación de Kruk, el grupo que dominó en Centenario fue el VI, que incluye organismos sin flagelos, con exoesqueleto de sílice. Éste estuvo acompañado por el VII, que involucra Chroococcales, estrategas k, de gran tamaño y volumen, que forman grandes colonias mucilaginosas. Según la clasificación de Reynolds, dominó el codón D, que incluye diatomeas de los géneros Synedra/Fragilaria, Nitzschia y algunas diatomeas céntricas; se asocia con aguas turbias poco profundas, es vulnerable al agotamiento de nutrientes y tolerante al lavado. Este grupo estuvo co-dominado por el codón $\mathrm{K}$, frecuente en hábitats poco profundos con columnas de agua enriquecidas y sensible a la mezcla profunda. En Centenario, la dominancia de diatomeas relativamente grandes podría explicarse por la ventaja competitiva que les confiere la turbulencia a pequeña escala (recirculado del agua) y, en algunos casos, la forma alargada de la célula, permitiéndoles ser más eficientes en la adquisición de nutrientes (Litchman et al. 2010, y citas en sus fuentes).

En este estudio se pudo evidenciar que los tres lagos urbanos estudiados, a pesar de tener una morfometría relativamente similar y encontrarse en la misma ciudad, difieren marcadamente en sus características limnológicas y en la estructura de sus comunidades fitoplanctónicas. Las condiciones limnológicas contrastantes determinan diferencias importantes en la estructura del fitoplancton delos tres lagos, principalmenteen sus especies dominantes, grupos funcionales, biomasa total de fitoplancton y tendencia al desarrollo de floraciones, apoyando las dos primeras hipótesis de este trabajo. El lago del Rosedal mostró ser el más eutrofizado, con una mayor turbidez y una clara dominancia de cianobacterias potencialmente tóxicas que alcanzan niveles propios de las floraciones. El lago de Regatas es el que presentó la mejor calidad del agua, con mayor transparencia y la menor biomasa de fitoplancton. El lago Centenario fue el de mayor conductividad y estuvo claramente dominado por diatomeas.

Las diferencias entre los lagos parecerían estar influenciadas por las medidas de manejo que en ellos se implementan, aunque para corroborar esta hipótesis sería necesario contar también con datos de otros componentes de la trama trófica. No obstante, los resultados de nuestras investigaciones mostraron que el lago de Regatas, con un recirculado de agua periódico y macrófitas sumergidas que se mantienen en buen estado por el corte periódico, es el que se mantiene en mejores condiciones. Por su parte, el lago de Parque Centenario se ve fuertemente influenciado por la entrada de agua subterránea y por eso presenta mayor conductividad y nitrógeno total, mientras que en el lago del Rosedal deberían implementarse medidas de restauración y mejores estrategias de manejo. Dado que estos lagos urbanos son muy usados con fines recreativos, es crucial mantener la calidad de las aguas a fin de prevenir el desarrollo de floraciones nocivas para el ser humano y para la fauna acuática.

Este estudio también puso de manifiesto que la diversidad funcional es una herramienta muy útil que permite caracterizar de forma adecuada a la comunidad fitoplanctónica de lagos urbanos, tal como lo revelaron los análisis multivariados basados en grupos funcionales, en los que se observó una clara separación de los tres ambientes, y similar a la observada en base a la composición de especies. En tal sentido, se concluye que el monitoreo de los lagos urbanos podría realizarse eficientemente evaluando los grupos funcionales.

Los lagos urbanos constituyen hotspots de biodiversidad en el entramado urbano y juegan un papel importante en la provisión de servicios ecosistémicos. Además, actúan como lugares de tránsito de especies animales al facilitar el movimiento de las mismas a través del paisaje (Céréghino et al. 2014; Hassall 2014). Por su rol ecológico, y ante los desafíos que plantea el cambio climático, se debe considerar una prioridad el control de la eutrofización de estos sistemas.

Agradecimientos Agradecemos a nuestros colegas M. Graziano y R. Lombardo por el asesoramiento en los análisis estadísticos. También deseamos expresar nuestro agradecimiento a los revisores del trabajo y a la editora (Dra. Mariana Meerhoff), cuyos aportes al trabajo fueron muy valiosos. Si bien no se contó con una fuente de financiamiento particular para estas investigaciones, agradecemos a la Facultad de Ciencias Exactas y Naturales de la Universidad de Buenos Aires, que permitió la utilización de infraestructura y equipamiento disponible en el laboratorio de Limnología de esta casa de estudios para llevarlas adelante. 


\section{REFERENCIAS}

APHA. 2005. Standard methods for the examination of water and wastewaters. American Public Health Association, Washington, USA.

Avigliano, L., A. Vinocur, G. Chaparro, and L. Allende. 2014. Influence of re-flooding on phytoplankton assemblages in a temperate wetland following prolonged drought. Journal of Limnology 73:247-262.

Backer, L. 2002. Cyanobacterial Harmful Algal Blooms (CyanoHABs): Developing a Public Health Response. Lake and Reservoir Management 18:20-31.

Britton, L., R. Averett, and R. Ferreira. 1977. Management of Urban Lakes. Water in the urban environment. Geological Survey Circular 601-K. U.S. Government Printing Office. USA.

Buenos Aires Ciudad. 2014. Ya se pueden disfrutar las mejoras en el Lago de Regatas. URL: www.buenosaires.gob.ar/ noticias/ya-se-pueden-disfrutar-las-mejoras-en-el-lago-de-regatas.

Carlson, R. 1977. A trophic state index for lakes. Limnology and Oceanography 22:361-369.

Céréghino, R., D. Boix, H-M. Cauchie, K. Martens, and B. Oertli. 2014. The ecological role of ponds in a changing world. Hydrobiologia 723:1-6.

Crossetti, L., V. Becker, L. de Souza Cardoso, L. Ribeiro Rodrigues, L. Silva da Costa, and D. da Motta-Marques. 2013. Is phytoplankton functional classification a suitable tool to investigate spatial heterogeneity in a subtropical shallow lake? Limnologica - Ecology and Management of Inland Waters 43:157-163.

Datos Acumar. URL: www.bdh.acumar.gov.ar:8081/bdh3/index_contenido.php.

Dignum, M., H. Matthijs, R. Pel, H. Laanbroek, and L. Mur. 2005. Nutrient Limitation of Freshwater Cyanobacteria. Tools to monitor phosphorus limitation at the individual level. Pp. 65-86 in J. Huisman, H. Matthijs and P. Visser (eds.). Harmful Cyanobacteria. Springer, The Netherlands.

Ehrenhaus, C., and M. Vigna. 2006. Changes in the phytoplankton of Lake Planetario after a restoration process. Darwiniana 44: 319-328.

Fazio, A., and I. O'Farrell. 2005. Phytoplankton and water quality in a shallow lake: a response to secondary salinization (Argentina). Wetlands 25:531-541.

Garay, D., and L. Fernández. 2013. Biodiversidad Urbana. Apuntes para un sistema de áreas verdes en la región metropolitana de Buenos Aires. Universidad Nacional de General Sarmiento. Buenos Aires, Argentina.

Gillett, N., M. Luttenton, and D. Steinman. 2015. Spatial and temporal dynamics of phytoplankton communities in a Great Lakes drowned river-mouth lake (Mona Lake, USA). Journal of Limnology 74:453-466.

Gómez, S., and N. Toresani. 1998. Región 3: PAMPAS. En P. Canevari, D. Blanco, E. Bucher, G. Castro and I. Davidson (eds.). Los Humedales de la Argentina: clasificación, situación actual, conservación y legislación. Wetlands International, Buenos Aires, Argentina.

Guo, Q., K. Ma, L. Yang, Q. Cai, and K. He. 2010. A comparative study of the impact of species composition on a freshwater phytoplankton community using two contrasting biotic indices. Ecological Indicators 10:296-302.

Hansen, A., and H. Márquez-Pacheco. 2012. Procedimiento para evaluar cargas internas de nutrientes en cuerpos de agua. Revista Mexicana de Ciencias Geológicas 1:265-275.

Hassall, C. 2014. The ecology and biodiversity of urban ponds. WIREs Water 1:187-206.

Hellström, D., U. Jeppsson, and E. Kärrman. 2000. A framework for systems analysis of sustainable urban water management. Environmental Impact Assessment Review 20:311-321.

Hillebrand, H., C. Dürselen, D. Kirschtel, U. Pollingher, and T. Zohary. 1999. Biovolume calculation for pelagic and benthic microalgae. Journal of Phycology 35:403-424.

Izaguirre, I., M. Boveda, and G. Tell. 1986. Dinámica del fitoplancton y características limnológicas en dos estanques de la ciudad de Buenos Aires. PHYSIS-A, Asociación Argentina de Ciencias Naturales, Sec. B 44:25-38.

Izaguirre, I., L. Allende, R. Escaray, J. Bustingorry, G. Pérez, and G. Tell. 2012. Comparison of morpho-functional phytoplankton classifications in human-impacted shallow lakes with different stable states. Hydrobiologia 698: 203-216.

Izaguirre, I., and R. Sinistro. 2012. Evaluación limnológica general y análisis de las comunidades planctónicas en el lago urbano de Parque Centenario de la Ciudad de Buenos Aires. Recomendaciones para su manejo. Datos no publicados. Informe técnico. Buenos Aires. Argentina.

Izaguirre, I., M. L. Sánchez, M. R. Schiaffino, I. O’Farrell, P. Huber, J. Zunino, L. Lagomarsino, and M. Mancini. 2015. Which environmental factors trigger the dominance of phytoplankton species across a moisture gradient of shallow lakes? Hydrobiologia 752:47-64.

Jeppesen, E., M. Søndergaard, T. L. Lauridsen, T. A. Davidson, Z. Liu, N. Mazzeo, C. Trochine, K. Özkan, H. S. Jensen, D. Trolle, F. Starling, X. Lazzaro, L. S. Johansson, R. Bjerring, L. Liboriussen, S. E. Larsen, F. Landkildehus, S. Egemose, and M. Meerhoff. 2012. Biomanipulation as a Restoration Tool to Combat Eutrophication: Recent Advances and Future Challenges. Advances in Ecological Research 47:411-488.

Koroleff, F. 1983. Simultaneous oxidation of nitrogen and phosphorus compounds by persulfate. Pp. 168-169 in K. Grosshoff, M. Eberhodt and K. Kremling (eds.). Methods of Seawater Analysis. Verlag Chemie, Wainherimer, Germany.

Kruk, C., V. Huszar, E. Peeters, S. Bonilla, L. Costa, M. Lürling, C. S. Reynolds, and M. Scheffer. 2010. A morphological classification capturing functional variation in phytoplankton. Freshwater Biology 55:614-627.

Kruk, C., A. Segura, L. Nogueira, C. Carballo, G. Martínez de la Escalera, D. Calliari, G. Ferrari, M. Simoens, J. Cea, I. 
Alcántara, P. Vico, D. Míguez, and C. Piccini. 2015. Herramientas para el monitoreo y sistema de alerta de floraciones de cianobacterias nocivas: Río Uruguay y Río de la Plata. Revista del Laboratorio Tecnológico del Uruguay. INNOTEC 10:23-39.

Litchman, E., P. de Tezanos Pinto, C. Klausmeier, M. Thomas, and K. Yoshiyama. 2010. Linking traits to species diversity and community structure in phytoplankton. Hydrobiologia 653:15-28.

Lürling, M., and F. van Oosterhout. 2013. Controlling eutrophication by combined bloom precipitation and sediment phosphorus inactivation. Water Research 47:6527-6537.

Lürling, M., E. Mackay, K. Reitzel, and B. M. Spears. 2016. Editorial - A critical perspective on geo-engineering for eutrophication management in lakes. Water Research 97:1-10.

Mantzouki, E., P. Visser, M. Bormans, and B. Ibelings. 2016. Understanding the key ecological traits of cyanobacteria as a basis for their management and control in changing lakes. Aquatic Ecology 50:333-350.

Margalef, R. 1978. Life-forms of phytoplankton as survival alternatives in an unstable environment. Oceanologica Acta 1:493-509.

Marker, A. F. H., A. Nusch, H. Rai, and B. Riemann. 1980. The measurement of photosynthetic pigments in freshwater and standardization of methods: conclusions and recommendations. Archive fur Hydrobiologie Beihandlung Ergebnisse del Limnologie 14:91-106.

Moss, B. 2007. The art and science of lake restoration. Hydrobiologia 581:15-24.

Murrel, M., and E. Lores. 2004. Phytoplankton and zooplankton seasonal dynamics in a subtropical estuary: importance of cyanobacteria. Journal of Plankton Research 26:371-382.

Naselli-Flores, L. 2008. Urban Lakes: Ecosystems at Risk, Worthy of the Best Care. Pp. 1333-1337 in M. Sengupta and R. Delwani (eds.). Proceedings of Taal 2007: The 12th World Lake Conference.

OCDE. 1982. Eutrophisation des eaux. Méthodes de surveillance, d'évaluation et de lute. Organisation de Coopération et de Développement Economiques, Paris, France.

Padisák, J., L. Crossetti, and L. Nasselli-Flores. 2009. Use and misuse in the application of the phytoplankton functional classification: a critical review with updates. Hydrobiologia 621:1-19.

Paerl, H., and J. Huisman. 2009. Climate change: a catalyst for global expansion of harmful cyanobacterial blooms. Environmental Microbiology Reports 1:27-37.

Paerl, H., N. Hall, and E. Calandrino. 2011. Controlling harmful cyanobacterial blooms in a world experiencing anthropogenic and climatic-induced change. Science of the Total Environment 409:1739-1745.

Phillips, G., N. Willby, and B. Moss. 2016. Submerged macrophyte decline in shallow lakes: What have we learnt in the last forty years? Aquatic Botany 135:37-45.

Pittaluga, G. 2014. Informe 77. Ola de calor en la última parte de octubre 2014. Eventos meteorológicos significativos. Departamento de Ciencias de la Atmósfera y de los Océanos. Universidad de Buenos Aires. Argentina.

Poikane, S., R. Portielje, M. van den Berg, G. Phillips, S. Brucet, L. Carvalho, U. Mischke, I. Ott, H. Soszka, and J. van Wichelen. 2014. Defining ecologically relevant water quality targets for lakes in Europe. Journal of Applied Ecology 51:592-602.

Qiu, D., Z. Wu, B. Liu, J. Deng, G. Fu, and F. He. 2001. The restoration of aquatic macrophytes for improving water quality in a hypertrophic shallow lake in Hubei Province, China. Ecological Engineering 18:147-156.

R-Core Team. 2016. R: A language and environment for statistical computing. R Foundation for Statistical Computing, Vienna, Austria. URL: www.R-project.org.

Reynolds, C. S. 1980. Phytoplankton assemblages and their periodicity in stratifying lake systems. Holarctic Ecology 3:141-159.

Reynolds, C. S. 1984. Phytoplankton periodicity: the interaction of form, functional and environmental variability. Freshwater Biology 14:111-142.

Reynolds, C. S. 1986. The ecology of freshwater phytoplankton. Cambrigde University Press, Cambridge, UK.

Reynolds, C. S. 1997. Vegetation Processes in the Pelagic: A model for Ecosystem Theory. Excellence in Ecology N 9 , Ecology Institute, Oldendorf, Germany.

Reynolds, C. S., V. Huszar, C. Kruk, L. Naselli-Flores, and S. Melo. 2002. Towards a functional classification of the freshwater phytoplankton. Journal of Plankton Research 24:417-428.

Ringuelet, R. A. 1962. Ecología acuática continental. Eudeba, Buenos Aires, Argentina.

Scasso, F., N. Mazzeo, J. Gorga, C. Kruk, G. Lacerot, J. Clemente, D. Fabián, and S. Bonilla. 2001. Limnological changes in a sub-tropical shallow hypertrophic lake during its restoration: two years of a whole-lake experiment. Aquatic Conservation: Marine and Freshwater Ecosystems 11:31-44.

Salmaso, N., and J. Padisák. 2007. Morpho-Functional Groups and phytoplankton development in two deep lakes (Lake Garda, Italy and Lake Stechlin, Germany). Hydrobiologia 578: 97-112.

Saad, J., M. Schiaffino, A. Vinocur, I. O'Farrell, G. Tell, and I. Izaguirre. 2013. Microbial planktonic communities of freshwater environments from Tierra del Fuego: dominant trophic strategies in lakes with contrasting features. Journal of Plankton Research 35:1220-1233.

Saad, M., and A. El-Karim. 2015. Survey to compare phytoplankton functional approaches: How can these approaches assess River Nile water quality in Egypt? Egyptian Journal of Aquatic Research 41:247-255.

Scarpati, O., and A. Capriolo. 2013. Sequías e inundaciones en la provincia de Buenos Aires (Argentina) y su distribución espacio-temporal. Investigaciones Geográficas, Boletín del Instituto de Geografía UNAM 82:38-51. 
Scheffer, M., S. H. Hosper, M. L. Meijer, B. Moss, and E. Jeppesen. 1993. Alternative equilibria in shallow lakes. Trends in Ecology and Evolution 8:275-279.

Scheffer, M., and E. Jeppesen. 2007. Regime Shifts in Shallow Lakes. Ecosystems 10:1-3.

Schuler, T., and J. Simpson. 2001. Introduction: Why urban lakes are different. Urban Lake Management. Watershed Protection Techniques 3:747-750.

Sinistro, R., S. Buitrago, A. Rennella, A. Mamani, and I. Izaguirre. 2013. Limnological changes in two shallow lakes from an urban reserve throughout a complete hydrological cycle: proposals for restauration and management. International Journal of Environment and Health 6:381-397.

Søndergaad, M., J. Jensen, and E. Jeppensen. 2001. Retention and internal loading of phosphorus in shallow, eutrophic lakes. The Scientific World 1:427-442.

ter Braak, C. J. F., and P. Smilauer. 2002. CANOCO Reference Manual and CanoDraw for Windows User's Guide: Software for Canonical Community Ordination (version 4.5). Microcomputer Power, New York. USA.

Timmerman, P., and R. White. 1997. Megahydropoiis: coastal cities in the context of global environmental change. Global Environmental Change 3:205-234.

Utermöhl, H. 1958. Zur Vervollkmnung der quantitativen Phytoplankton-Methodik, Mitteilungen, Internationale Vereiningung fur Theorestische und Angewandte Limnologie 9:1-38.

Venrick, E. L. 1978. How Many Cells to Count? Paris, Unesco.

Waajen, G., E. Faassen, and M. Lürling. 2014. Eutrophic urban ponds suffer from cyanobacterial blooms: Dutch examples. Environmental Science and Pollution Research 21:9983-9994.

Waajen, G., F. van Oosterhout, G. Douglas, and M. Lürling. 2016. Geo-engineering experiments in two urban ponds to control eutrophication. Water Research 97:69-82.

Wang, Z., D. Li, H. Qin, and Y. Li. 2012. An integrated method for removal of harmful cyanobacterial blooms in eutrophic lakes. Environmental Pollution 160:34-41.

WHO. 2003. Guidelines for Safe Recreational Water Environments. Volume 1, Coastal and Fresh Waters. World Health Organization. 